\title{
The E3 ubiquitin ligase Siah1 regulates adrenal gland organization and aldosterone secretion
}

\author{
Marzia Scortegagna, ${ }^{1}$ Annabel Berthon, ${ }^{2}$ Nikolaos Settas, ${ }^{2}$ Andreas Giannakou, ${ }^{2}$ \\ Guillermina Garcia, ${ }^{1}$ Jian-Liang Li, ${ }^{1}$ Brian James, ${ }^{1}$ Robert C. Liddington, ${ }^{1}$ José G. Vilches-Moure, ${ }^{3}$ \\ Constantine A. Stratakis, ${ }^{2}$ and Ze'ev A. Ronai ${ }^{1,4}$ \\ 'Sanford Burnham Prebys Medical Discovery Institute, La Jolla, California, USA. ${ }^{2}$ Section on Endocrinology and Genetics, \\ Eunice Kennedy Shriver National Institute of Child Health and Human Development, NIH, Bethesda, Maryland, USA. \\ ${ }^{3}$ Department of Comparative Medicine, Stanford University Medical Center, Stanford, California, USA. ${ }^{4}$ Technion Integrated \\ Cancer Center, Faculty of Medicine, Technion, Israel Institute of Technology, Haifa, Israel.
}

Primary and secondary hypertension are major risk factors for cardiovascular disease, the leading cause of death worldwide. Elevated secretion of aldosterone resulting from primary aldosteronism (PA) is a key driver of secondary hypertension. Here, we report an unexpected role for the ubiquitin ligase Siah1 in adrenal gland development and PA. Siah1a ${ }^{-/-}$mice exhibit altered adrenal gland morphology, as reflected by a diminished X-zone, enlarged medulla, and dysregulated zonation of the glomerulosa as well as increased aldosterone levels and aldosterone target gene expression and reduced plasma potassium levels. Genes involved in catecholamine biosynthesis and cAMP signaling are upregulated in the adrenal glands of Siah1a $a^{-/-}$mice, while genes related to retinoic acid signaling and cholesterol biosynthesis are downregulated. Loss of Siah1 leads to increased expression of the Siah1 substrate PIAS1, an E3 SUMO protein ligase implicated in the suppression of LXR, a key regulator of cholesterol levels in the adrenal gland. In addition, SIAH1 sequence variants were identified in patients with PA; such variants impaired SIAH1 ubiquitin ligase activity, resulting in elevated PIAS1 expression. These data identify a role for the Siah1-PIAS1 axis in adrenal gland organization and function and point to possible therapeutic targets for hyperaldosteronism.

Conflict of interest: The authors have declared that no conflict of interest exists.

Submitted: August 28, 2017 Accepted: October 19, 2017 Published: December 7, 2017

\section{Reference information:} JCI Insight. 2017;2(23):e97128. https://doi.org/10.1172/jci. insight. 97128 .

\section{Introduction}

Hypertension is one of the most important drivers of cardiovascular disease. Primary hypertension, which accounts for about $90 \%$ of cases, is idiopathic, whereas secondary hypertension is caused by an underlying medical condition. The leading cause of secondary hypertension - accounting for 5\%-13\% of cases - is primary aldosteronism (PA), which is characterized by aberrant secretion of aldosterone and is unresponsive to the renin-angiotensin system and sodium homeostasis (1-3). Although PA has several causes, about a third of cases are due to aldosterone-producing adenomas. Approximately $95 \%$ of cases are sporadic and another $5 \%$ are familial. Genetic mutations in a number of genes, including those encoding ion channels (KCNJ5, ATP1A1, ATP2B3, CACNA1D, and CACNA1H) and proteins involved in cell-cell and protein-protein ( $A R M C 5, C T N N B 1)$ interactions, have been implicated in PA Finally, perturbations in epigenetic regulatory mechanisms have also been associated with overproduction of aldosterone $(4,5)$.

Aldosterone and other steroid hormones primarily produced in the adrenal glands and gonads play key roles in mammalian physiology, including regulation of ion balance and blood glucose levels; fat, protein, and carbohydrate metabolism; inflammation and immunity; and sexual differentiation. Accordingly, steroid hormones are tightly regulated by transcriptional and posttranslational mechanisms, including phosphorylation, acetylation, ubiquitination, and sumoylation (6-9). Fine-tuning of steroid hormone levels, which is essential for their spatiotemporal regulation, is mediated by the ubiquitin-proteasome (UBP) and small ubiquitin-like modifier (SUMO) pathways (10). Indeed, adrenal cortex development and steroidogenesis are controlled by the UBP-SUMO-dependent modification of regulatory transcription factors, such as SF1, WT1, GLI3, SALL1, and DAX1 (11-14). 
The RING finger E3 ubiquitin ligases Siah1 and Siah2 are conserved from Drosophila to vertebrates (15). Siah1/2 ligases target proteins for UBP-mediated degradation, implicating them in the control of many central regulatory processes, including hypoxia (via control of prolyl-hydroxylases 1 and 3) (16), endoplasmic reticulum stress (via ATF4) (17), cell-cycle progression and cell junction integrity (via ASPP2) (18), mitochondrial dynamics (via AKAP121) (19), and intracellular signaling (via MAPK) (20). Mouse embryonic fibroblasts derived from mice lacking Siah1a, one of two forms of Siah1 in the mouse (1a/1b), show no marked changes in growth or cell-cycle regulation (21). However, Siah1a ${ }^{-1-}$ mice display a number of defects, such as postnatal growth retardation, osteopenia, sterility, and premature death, although growth hormone and gonadotropin levels appear normal in these mice $(21,22)$. Here, we identify that Siah1 regulates adrenal gland structure and function in the development of PA.

\section{Results}

Siah $1 a^{-1-}$ mice display altered adrenal gland morphology, with a diminished $X$-zone and enlarged medulla. Previous studies detected no abnormalities in the vital organs or in the levels of gonadotropins and growth hormone in Siahl $1 a^{-1-}$ mice (21), even though growth retardation and increased mortality was observed. Using Sia$h 1 a^{-/-}$mice in the $129 \mathrm{sv}$ genetic background, we observed premature death with survival rate as previously reported, where no Siah1a ${ }^{-1-}$ mice survived beyond 30 days. While body weight at embryonic day 18.5 was normal, a significant decrease in weight was observed in Siah1 $a^{-/-}$mice at postnatal day 1.5, with a further decrease at postnatal day 21 (Supplemental Figure 1A; supplemental material available online with this article; https://doi.org/10.1172/jci.insight.97128DS1). Analysis of young (21-day-old) Siah1a ${ }^{-/-}$mice revealed marked changes in the morphology of the adrenal glands (Figure 1, A-C) but not in their size

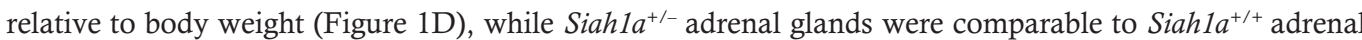
glands (data not shown). The adrenal glands of Siah1 ${ }^{-/-}$mice (21-day-old females and males) contained a much diminished X-zone (Figure 1, A-C) compared with glands from WT littermates. Correspondingly, the expression of 20- $\alpha$-hydroxysteroid dehydrogenase (20- $\alpha \mathrm{HSD})$, an X-zone marker, was reduced in Siah1 $1 a^{-1-}$ mice both at the protein and RNA level (Figure 1, E and F). Similarly, expression of phosphatidylinositol-4-phosphate 3-kinase (PIK3C2 $\gamma$ ), another X-zone marker, was reduced by approximately $70 \%$ (Figure 1F). Siah1 expression in the adrenal gland was confirmed by qPCR analysis (Supplemental Figure 1B), and in situ hybridization indicated that Siah1a was expressed in the zona glomerulosa, zona fasciculata, and medulla in both 15-day-old embryos and 21-day-old mice (Supplemental Figure 1C), substantiating a role for Siah1a in adrenal gland development. Quantification of the area occupied by tyrosine hydroxylase-positive (TH-positive) cells versus the total area of the adrenal gland revealed an enlarged medulla in Siah1 1 ${ }^{-1-}$ mice (Figure 2, A and B). Accordingly, a significant (3-fold) increase of TH mRNA expression was observed in Siah1 $a^{-1-}$ mice (Figure 2C). Given the increased TH mRNA expression and the observed enlarged medulla, we tested the level of catecholamine in plasma. A significant increase in adrenaline was observed in plasma from Siah1 $a^{-/-}$mice, along with an increase in noradrenaline (Figure 2, D and E).

Glomerulosa zonation is dysregulated in Siah1a ${ }^{-1}$ mice. To further probe the role of Siah1a in adrenal gland development, we monitored organization of the zona glomerulosa and zona fasciculata in 21-day-old Siahla ${ }^{-1}$ and WT mice by staining for expression of Cyp11b2 (aldosterone synthase, expressed in the zona glomerulosa), which is required for aldosterone synthesis, and Cyp11b1 (11- $\beta$-hydroxylase, expressed in the zona fasciculata). Although the expression pattern of Cyp11b1 was spatially similar in the adrenal glands of Siah1 $a^{-/-}$and WT mice, staining was more intense in the Siah1 $a^{-1}$ glands (Figure 3A). In contrast, while Cyp11b2 expression was limited to the zona glomerulosa in WT adrenal glands, it was present in both the zona glomerulosa and zona fasciculata of Siah1 $1 a^{-/-}$mice (Figure 3A). To substantiate the altered zonation in Siah1 $1 a^{-1-}$ mice, we examined expression of disabled homolog 2 (Dab2), another marker of the zona glomerulosa. Indeed, as observed for Cyp11b2, Dab2 expression extended from the zona glomerulosa to the outer zona intermedia and the zona fasciculata of Siah1 $1 a^{-1-}$ mice but was detected only in the zona glomerulosa of WT mice (Figure 3B).

Cyp11b1 and Cyp11b2 mRNA levels were elevated in the adrenal glands of Siah1 $a^{-1-}$ mice compared with WT littermates, (Figure 3C). Correspondingly, plasma aldosterone levels were also increased 2-fold (Figure 3D). Of note, Dab2 was previously shown to elevate both Cyp11b2 expression and aldosterone secretion in H295R cells, which could explain the observed increase of Cyp11b2 expression in Siah1 $a^{-/-}$mice, at least in part (23). A slight increase in plasma corticosterone levels was noted in the Siah1 $a^{-/-}$mice, although the difference was not statistically significant (Figure 3D). ACTH stimulation did not alter the level of plasma corticosterone (Supplemental Figure 2A). Further, under normal fed conditions, the level of plasma ACTH 


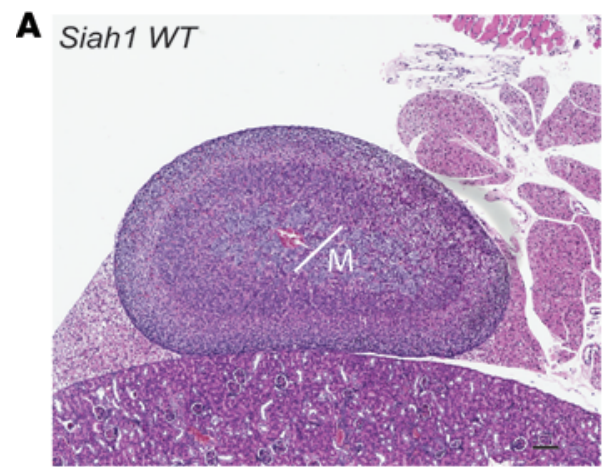

B

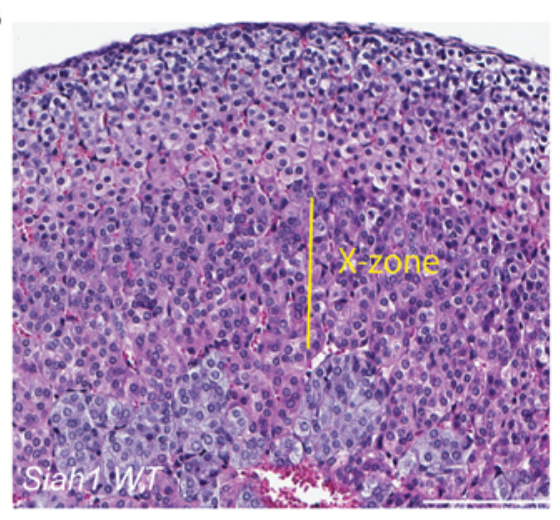

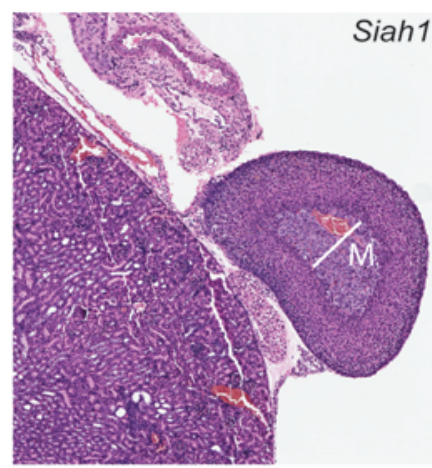

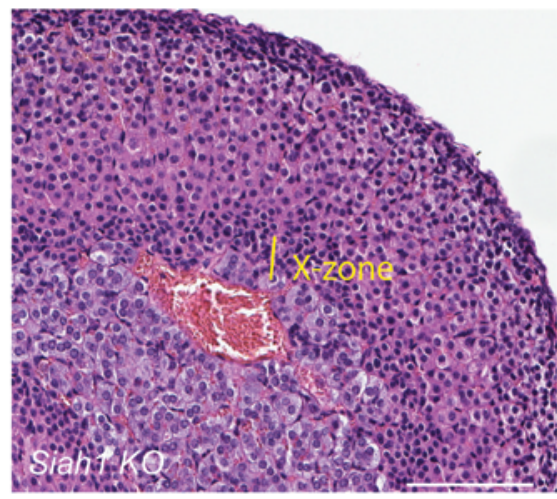

C

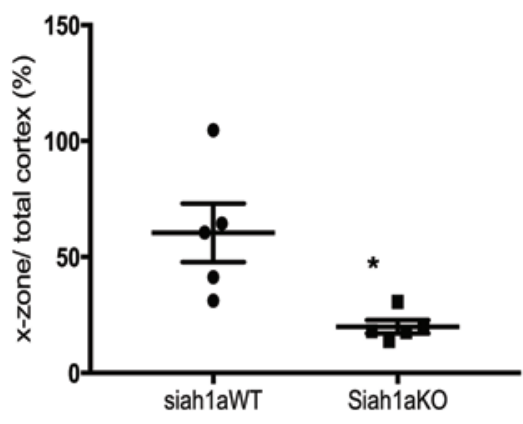

D

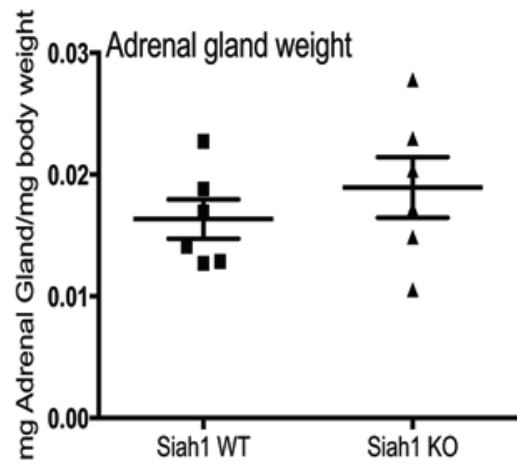

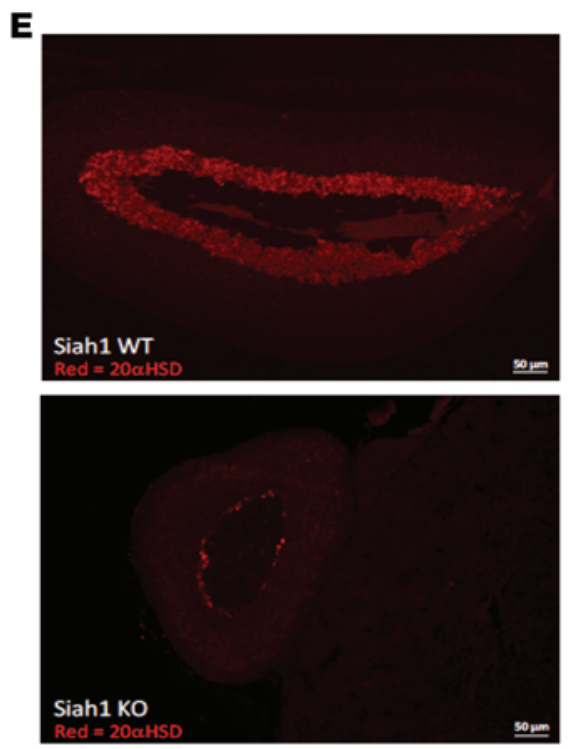
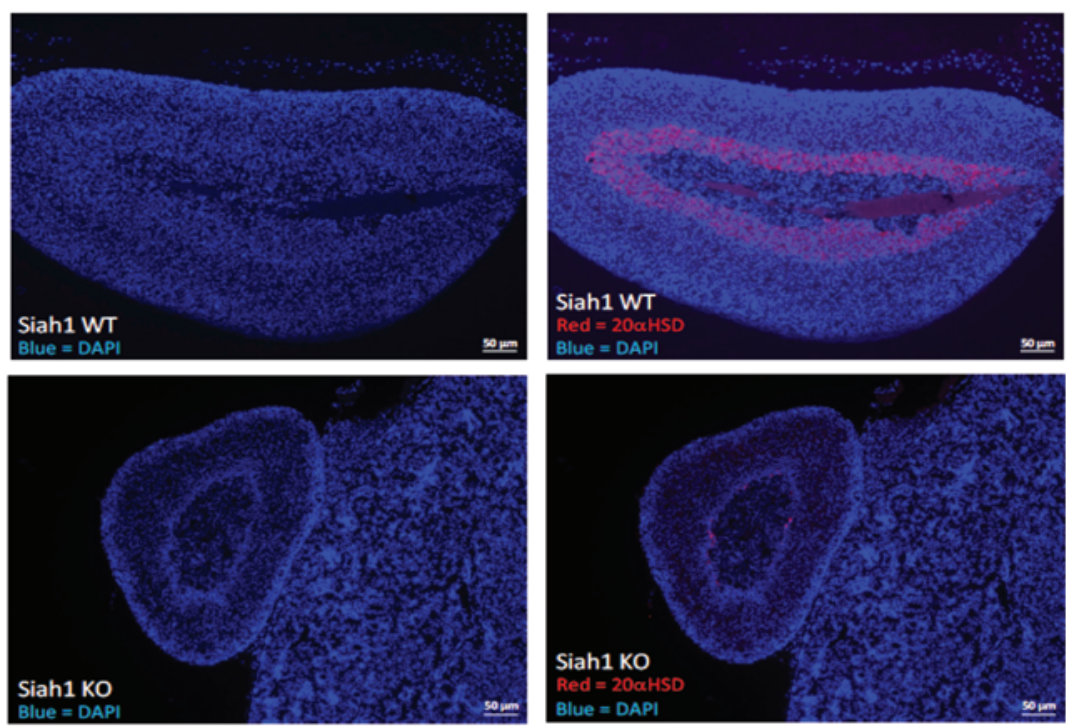

$\mathbf{F}$
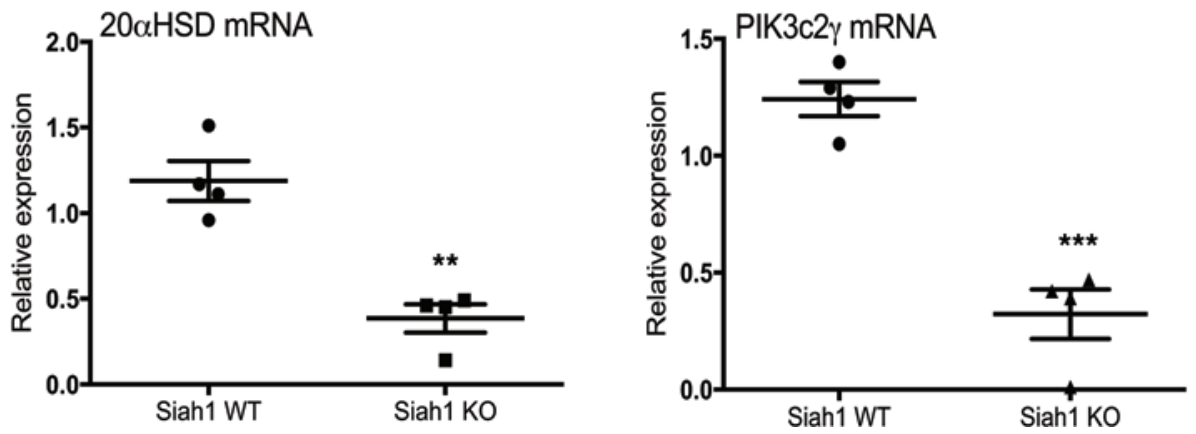

Figure 1. Altered morphology of the adrenal glands with diminished X-zone in Siah1a-/- mice. (A-C) H\&E staining of 21-day-old WT adrenal glands and Siah1a ${ }^{-1-}\left(\right.$ Siah1a KO) mice at low (A) and high (B) magnification and quantitation of the X-zone (C). Five mice were analyzed for X-zone quantitation. ${ }^{*} P<$ 0.05 , compared with WT. Glands from Siah1a KO mice show aberrant morphology, with a smaller X-zone. The white and yellow lines indicate the medulla 
(M) and X-zone, respectively. Scale bar: $100 \mu \mathrm{m}$. (D) Adrenal gland weights relative to body weights of 21-day-old Siah1a ${ }^{-1-}$ mice and WT littermates ( $n=6$ ). (E) Representative immunofluorescence for the X-zone marker, 20- $\mathrm{HSSD}$, in 21-day-old-WT and Siah1a ${ }^{-/-}$adrenals. Although 20- $\alpha \mathrm{HSD}$ expression is found in both WT and KO adrenals, its expression is significantly reduced in Siah1a ${ }^{-1-}$ mice. (F) qPCR analysis of 20 $\alpha H S D$, and PIK3C2 $\gamma$ expression in the adrenal glands of 21-day-old Siah1a ${ }^{-/-}$and WT mice $(n=4) .{ }^{*} P<0.005,{ }^{* *} P<0.0005$, compared with WT. Data are shown as mean \pm SEM. Statistical analysis was performed using $t$ test.

was similar in both Siah1a ${ }^{-/-}$and WT mice, excluding other potential causes for hyperaldosteronism, (Supplemental Figure 2B). Notably, the ratio between aldosterone and renin activity was elevated in Siahla ${ }^{-1-}$ plasma, supporting primary hyperaldosteronism in the Siah1 $a^{-/-}$mice (Supplemental Figure 2C).

To analyze the role of Siah1 in adrenal gland development, we determined the expression pattern of Dab2 and Cyp11b1 at gestational day 15. While the expression patterns of TH and Cyp11b1 were spatially similar in the adrenal glands of Siah1 $a^{-/-}$and WT mice (Supplemental Figure 2E), an increased number of Dab2-positive cells, which exhibited a more intense staining, was seen in the adrenal gland of Siah1 $a^{-1}$ mice (Supplemental Figure 2D). These findings suggest that Siah1 regulates adrenal gland zonation and aldosterone secretion.

Increased expression of aldosterone target genes in Siah1a ${ }^{-1-}$ mice coincides with reduced plasma $K^{+}$levels. Since aldosterone manifests its major physiological effects in the epithelial cells of renal nephrons, we investigated possible changes in the expression of the aldosterone target genes serum/glucocorticoid-regulated kinase 1 (SGK1) and epithelial $\mathrm{Na}^{+}$channel (eNAC $\alpha$ ) in the kidneys $(24,25)$. SGK1 controls eNAC $\alpha$ protein expression by phosphorylating and inactivating Nedd4-2, an E3 ubiquitin ligase that regulates eNAC $\alpha$ stability (26). We observed a 3-fold increase in SGK1 mRNA levels and a 2-fold increase in SCNN1a (eNAC $\alpha$ ) mRNA levels in the kidneys of Siah1 $a^{-/-}$mice compared with those of WT littermates (Figure 4, A and B), while the increase observed for eNAC $\beta$ and eNAC $\gamma$ mRNA was minimal (Supplemental Figure 3). Immunohistochemistry analysis confirmed an increase in SGK1 and eNAC $\alpha$ protein expression in distal convoluted tubules, in connecting tubules, and in the collecting ducts of kidneys in Siahla $a^{-1-}$ mice compared with WT mice (Figure 4, C and D). eNAC $\alpha$ activity plays an important role in $\mathrm{Na}^{+} / \mathrm{K}^{+}$homeostasis by promoting $\mathrm{Na}^{+}$retention and $\mathrm{K}^{+}$secretion. In keeping with the observed increase in eNAC $\alpha$ expression, we found that levels of $\mathrm{K}^{+}$were markedly decreased in the plasma of Siah1a ${ }^{-1-}$ mice compared with WT mice (Figure 4E). Thus, Siah1a is required for maintenance of normal plasma $\mathrm{K}^{+}$levels through regulation of aldosterone levels and expression of aldosterone target genes. These data suggest that perturbation in Siah1a function may contribute to hypertension and electrolyte imbalance.

Genes related to retinoic acid signaling and cholesterol biosynthesis are dysregulated in the adrenal glands of Sia$h 1 a^{-/-}$mice. To further examine the involvement of Siah1a in regulating adrenal gland function, we performed unbiased gene expression analysis of the adrenal glands of Siah1 $a^{-1-}$ and WT mice under basal conditions and following ACTH stimulation (Figure 5A and Supplemental Figure 4A). Pathway analysis of the modulated genes identified those involved in cholesterol biosynthesis and retinoic acid signaling (liver and retinoid X receptors [LXR/RXR]) to be among the most downregulated, while catecholamine biosynthesis and CAMP-mediated signaling pathway genes were among the most upregulated genes in Sia$h 1 a^{-/-}$adrenal glands compared with WT adrenal glands (Figure 5A). A significant increase of adrenaline in plasma from Siahla ${ }^{-1-}$ mice, compared with WT mice, suggests an increase in catecholamine biosynthesis (Figure 2D). Changes in the expression of individual genes involved in the LXR/RXR pathway, cholesterol biosynthesis, and cAMP-mediated signaling were confirmed by qRT-PCR (Supplemental Figure 4, B-D). The decreased expression of $A p o E$ mRNA, which is involved in cholesterol storage, and the increased expression of SCARB1 mRNA, which is involved in cholesterol uptake (Supplemental Figure 4, B and D), suggest that intracellular cholesterol levels may be increased in the adrenal glands of Siah1 $1 a^{-/}$mice. Induction of free cholesterol and cholesteryl esters would be expected to suppress endogenous cholesterol synthesis: indeed, this was observed in the adrenal glands of Siah1 $a^{-/-}$mice, as shown by RNA-seq analysis, and confirmed by qPCR assays (Supplemental Figure 4, B-D). Furthermore, an increase in the number and size of neutral lipids was observed in adrenal glands of Siah1 $1 a^{-1-}$ mice, compared with WT mice, using Oil Red O staining (Supplemental Figure 4E). Consistent with these observations, increased neutral lipid levels and production of steroid hormones have been reported in the adrenal glands of mice lacking LXR (27).

PIAS1 is upregulated in the adrenal glands of Siah1 $a^{-1-}$ mice. The E3 SUMO protein ligase PIAS1 was previously shown to be a substrate of Siah $1 / 2$ and to undergo UBP-dependent degradation in the nucleus $(28,29)$. PIAS1 has also been implicated in the suppression of LXR in a Siah1-dependent manner $(28,29)$. 
A
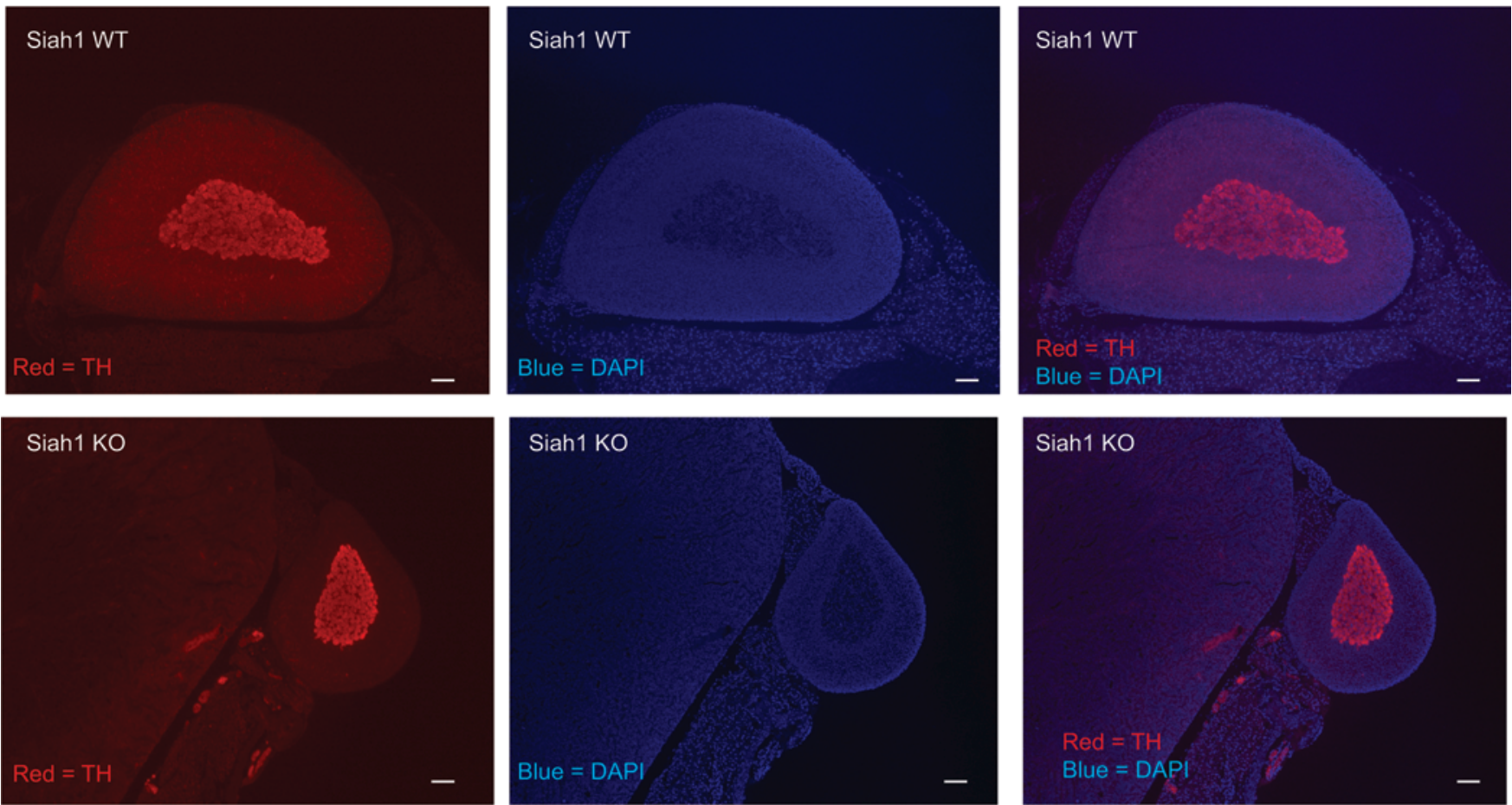

B

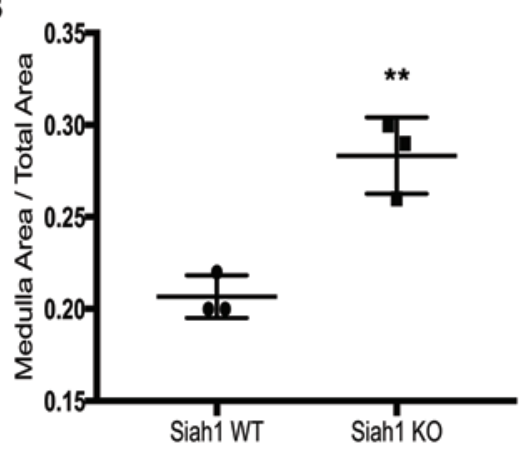

D

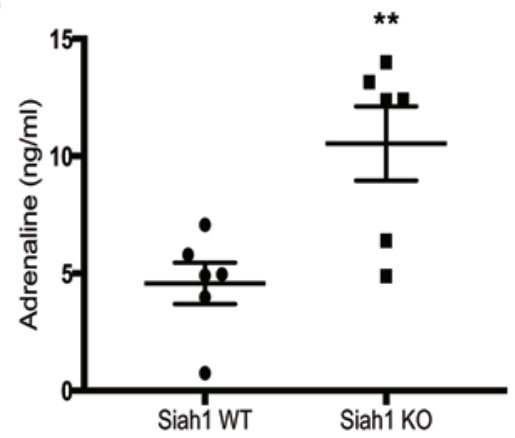

C

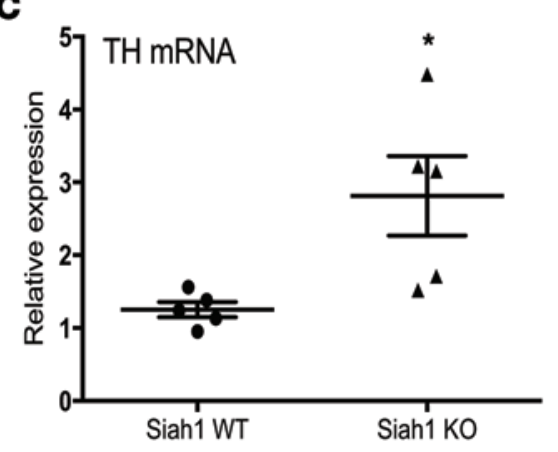

E

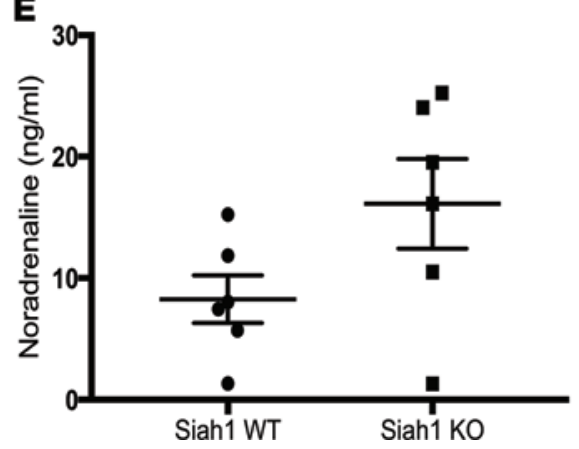

Figure 2. Enlarged medulla and increased adrenaline in plasma of Siah1a ${ }^{-/-}$mice. (A) Representative immunofluorescence for the medulla marker, TH, in 21-day-old WT and Siah1a $a^{--}$adrenals. Scale bar: $100 \mu \mathrm{m}$. (B) Three mice were analyzed for medulla quantitation. ${ }^{* *} P<0.005$, compared with WT. Glands from Siah1a $a^{-1-}$ mice show a larger medulla. (C) qPCR analysis of TH expression in the adrenal glands of 21-day-old Siah1a ${ }^{-1-}$ and WT mice $(n=5) .{ }^{*} P<0.05$, compared with WT. (D) Adrenaline and (E) noradrenaline levels in the plasma of WT and Siah1a ${ }^{-1-}$ mice measured by ELISA $(n=6)$ ${ }^{*} P<0.005$, compared with WT. Data are shown as mean \pm SEM. Statistical analysis was performed using $t$ test. 
A
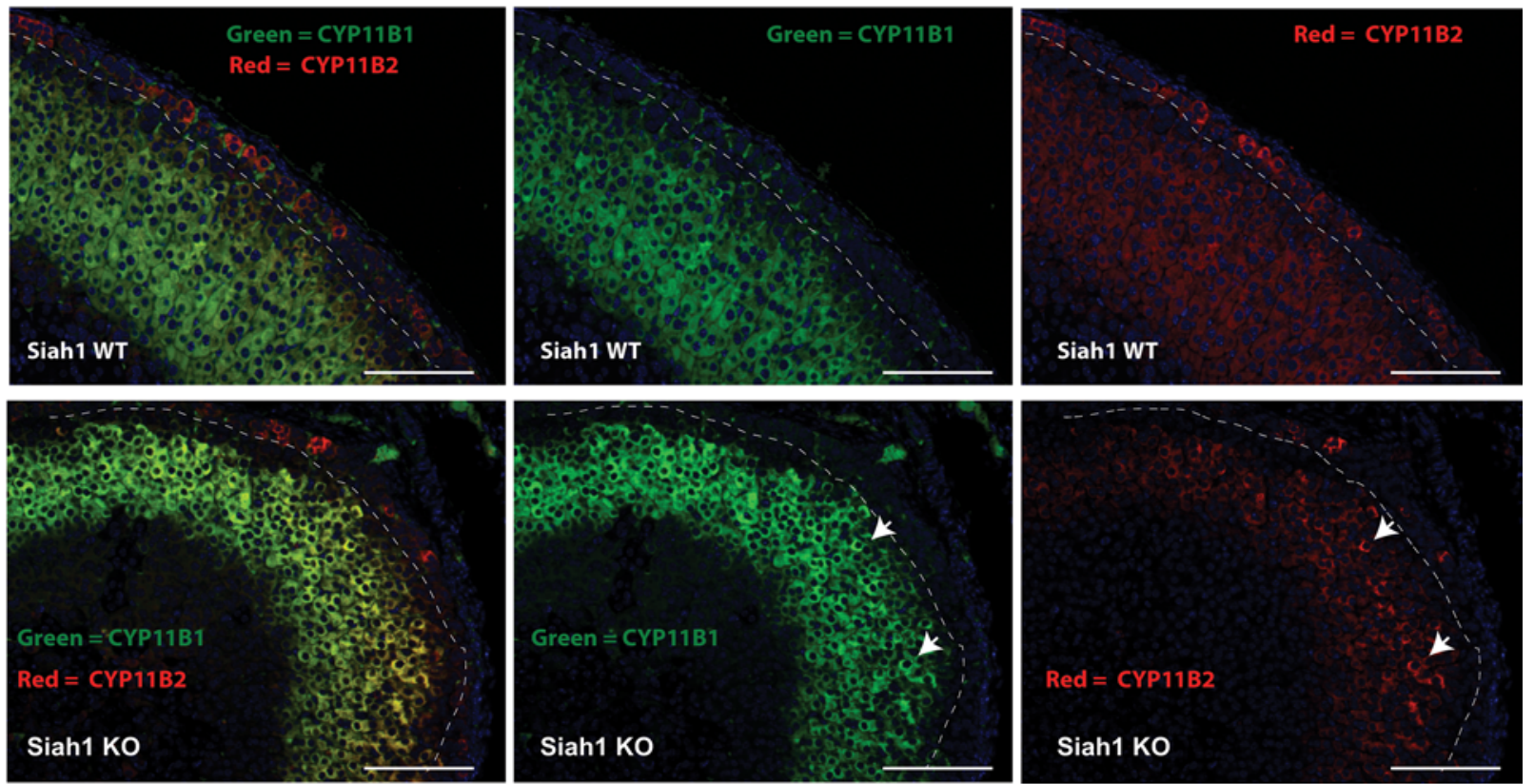

B
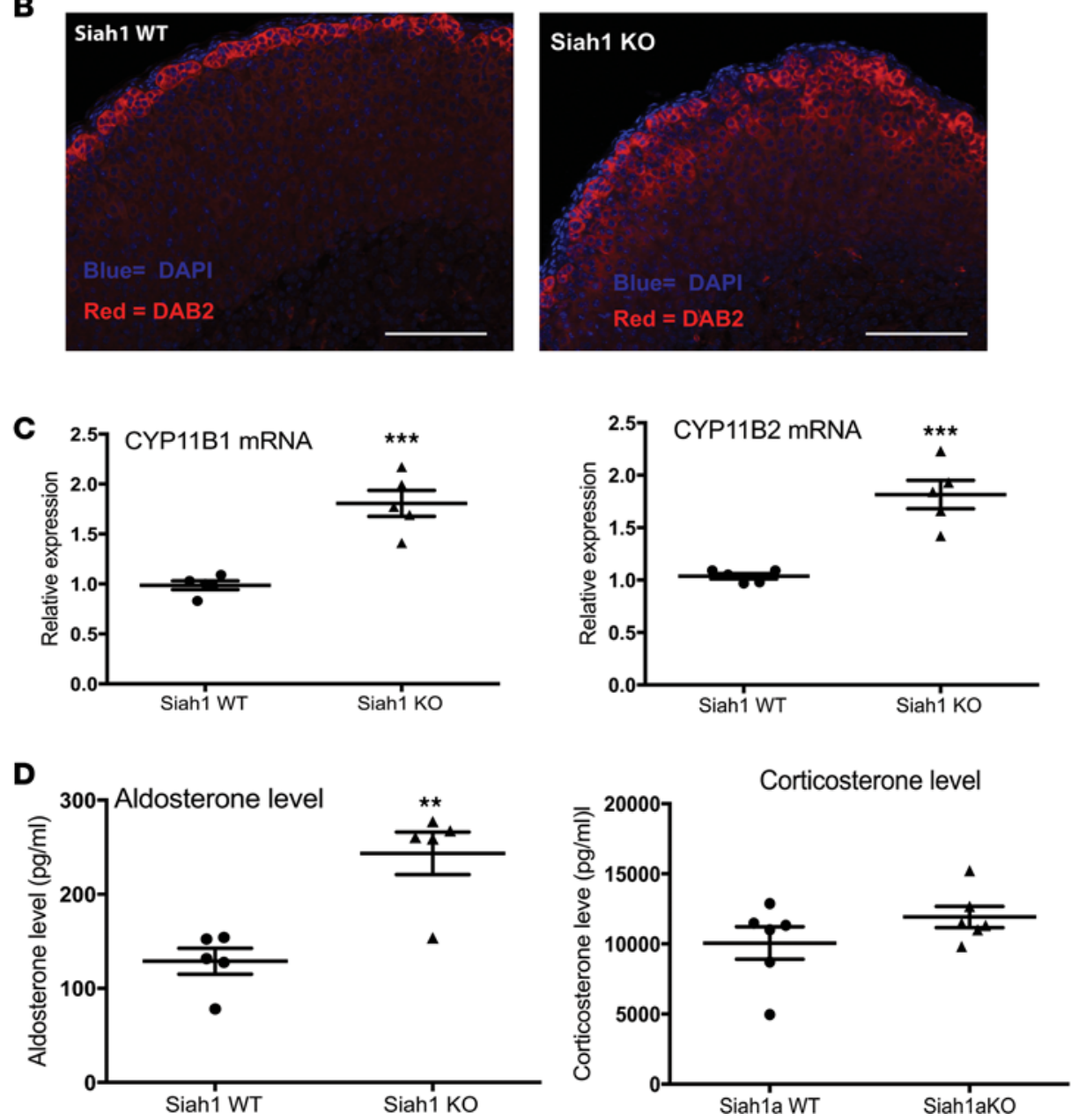

Figure 3. Dysregulation of zona glomerulosa cells and increased aldosterone secretion in Siah1a-/- mice. (A) Sections of adrenal glands costained for Cyp11b1 (green) and Cyp11b2 (red). Cyp11b1 staining is seen in the zona fasciculata of both WT and Siah1a ${ }^{-1}$ (KO) mice. Cyp11b2 staining is restricted to the zona glomerulosa of the outer adrenal cortex in WT mice but extends from the zona glomerulosa to the inner parts of the adrenal cortex in Siah1a KO mice. White dashed lines separate zona glomerulosa from zona fasciculata. White arrows indicate Cyp11b1 and Cyp11b2 colocalization. Scale bar: $100 \mu$ m. (B) 
Staining of DAB2 (red) and nuclei (DAPI, blue) in sections of adrenal glands. DAB2 staining is restricted to the outer adrenal cortex of WT mice but extends to the inner parts of the adrenal cortex in Siah1a KO mice. Images shown in $\mathbf{A}$ and $\mathbf{B}$ are representative of images from 4 different mice per group. Scale bar: $100 \mu \mathrm{m}$. (C) qPCR analysis of Cyp11b1 and Cyp11b2 mRNA in the adrenal glands $(n=5) .{ }^{* *} P<0.0005$, compared with WT. (D) Aldosterone and corticosterone levels in the plasma of WT and Siah1a KO mice measured by ELISA ( 5 mice for aldosterone and 6 mice for corticosterone levels). ${ }^{* *} P<0.005$, compared with WT. Data are shown as mean \pm SEM. Statistical analysis was performed using $t$ test.

PIAS1, which was implicated in the control of cholesterol levels in the adrenal gland has also been shown to affect lipogenesis and inflammatory signaling (28-30). PIAS1 increases the expression of both CYP11B1 and CYP11B2 mRNA by enhancing sumoylation of the transcription factor SF1 and activating the transcription factor COUP-TFI, respectively (31-33). Therefore, we investigated PIAS1 expression in Siah1 $1 a^{-1-}$ mice. PIAS1 expression in the adrenal glands of WT mice was highest in the zona glomerulosa, as detected by immunostaining (Figure 5B), consistent with previous reports (31). Strikingly, however, PIAS1 was expressed throughout the adrenal glands of Siah1 $1 a^{-1-}$ mice, and this was accompanied by increased nuclear localization (Figure 5B). This increase in expression was confirmed by immunoblot analysis (Figure 5C). Interestingly, levels of PIAS1 mRNA were the same in Siah1a ${ }^{-/-}$and WT mice, substantiating the increase in protein expression was posttranslationally regulated (Supplemental Figure 4F). To confirm that Siah1 controls the stability of PIAS1 protein, we performed pulsechase experiments with 293T cells cotransfected with Siah1 and PIAS1; as expected, the degradation of PIAS1 was accelerated in the cells coexpressing Siah1 (Figure 5D). In the presence of Siah1, about $25 \%$ of PIAS1 protein was degraded within 2 hours, compared with 5 hours in the absence of Siah1 (Figure 5D). Collectively, these observations suggest that the expression of CYP11B2 and CYP11B1 and, potentially, cholesterol biosynthesis in the adrenal glands, are regulated by Siah1a via its control of PIAS1 stability. Since $\beta$-catenin was previously identified as a Siah substrate and given its role on adrenal gland function, we determined possible changes in $\beta$-catenin expression in adrenal glands of Siah $1 a^{-/-}$and WT mice. $\beta$-Catenin expression in adrenal glands of Siah1 $a^{-/-}$mice appeared to be less membranal and more cytoplasmic/nuclear (Supplemental Figure 4G). $\beta$-Catenin is known to induce angiogenesis by upregulating VEGFA mRNA. Indeed, blood vessels appeared more dilated in adrenal glands derived from Siahl $a^{-/-}$mice (Supplemental Figure 4H); however the mRNA levels of VEGFA, $L E F 1$, and Axin2, $\beta$-catenin transcriptional targets, were unchanged (Supplemental Figure 4I), suggesting that either different transcriptional targets are affected or changes elicited upon altered localization of $\beta$-catenin may not be transcriptional.

The adrenal glands of patients with primary hyperaldosteronism express a mutant form of Siah1 and elevated expression of PIAS1. The phenotype identified in the Siah1 $a^{-/-}$mice resembles that of patients with hyperaldosteronism; therefore, we investigated the possibility that the adrenal glands of patients with PA might display genetic or epigenetic alterations in Siah1 and PIAS1. Analysis of the SIAH1 gene was carried out in 81 patients, including 60 with PA and 21 with combined glucocorticoid and mineralocorticoid hormone secretion. The clinical data of these patients are presented in Table 1. Among these patients, 7 SIAH1 genetic variants were identified; 2 were missense and 5 were synonymous; loss of heterozygosity was identified in only one of the tumors (Table 2).

Further characterization was performed on one of the SIAH1 missense variants (NM_003031), which harbored a substitution in amino acid 251 from Ile to Leu. This residue is located on a helix-loophelix motif that lies on the surface of the substrate-binding domain (SBD) and spans from the dimer interface to the linker region between $\mathrm{Zn}$-fingers 1 and 2 (ZnF-1 and $\mathrm{ZnF}-2$ ) (Figure 6A). The side chain of Ile 251 is mostly buried, although one face is solvent-exposed and lines a prominent pocket (part of the SBD also serves as a site for small-molecule inhibitors, based on analyses of its similarly structured homolog, Siah2) (34). We previously showed that mutations in either the adjoining helical residue, met 252, or an adjacent "KYD" loop caused loss of function (35). Both of these 2 elements are surface-exposed, forming a quasicontiguous patch across the dimer interface. Thus, it is plausible that loss of direct interactions with substrate affects their ability to associate and degrades their substrate(s). Nonetheless, the structural basis for a strong loss-of-function effect for the buried and conservative I251L mutant is less obvious. Comparison of 3 different crystal forms of SIAH1 suggests that, in each case, ZnF-2 (and, by extension, the N-terminal RING domain - not shown) adopts distinct locations related by a relative rotation (of up to $30^{\circ}$ ) about a single axis (" $R$ " in Figure 6A) with respect to the SBD. This observed variation points to high-mobility/multiple conformations available to the ZnF-2-RING moiety. Of 
A

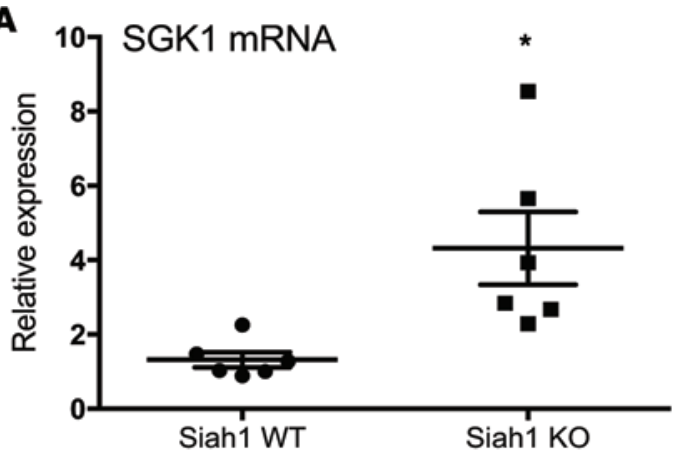

C

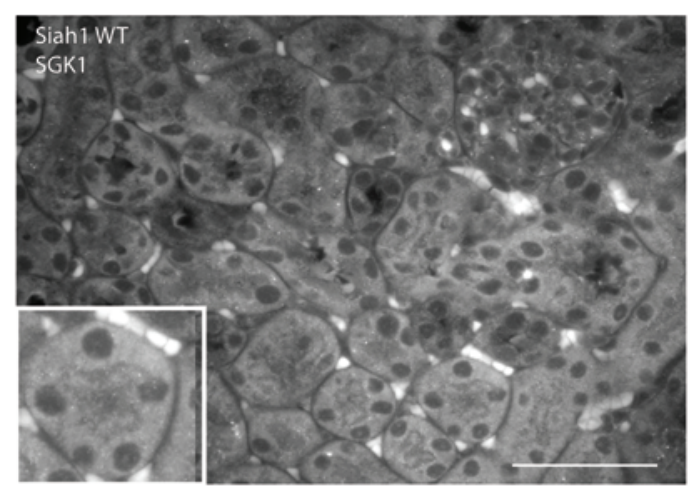

D

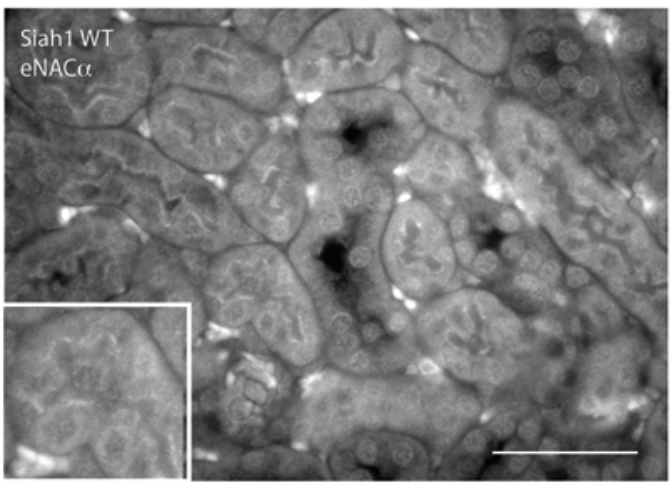

E

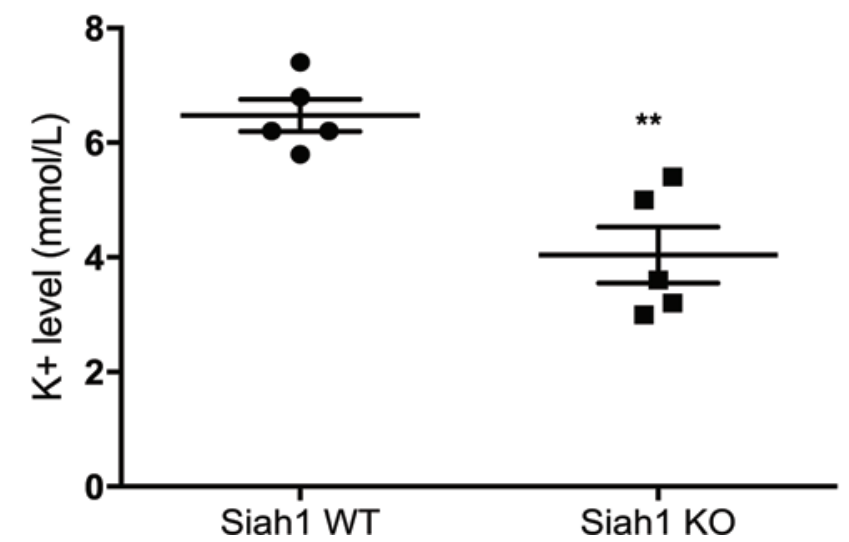

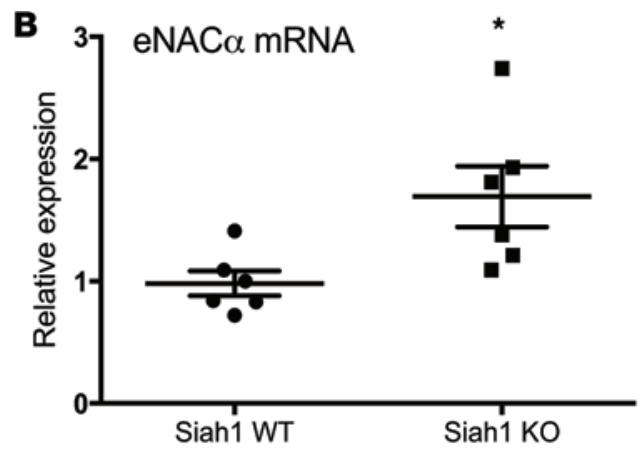
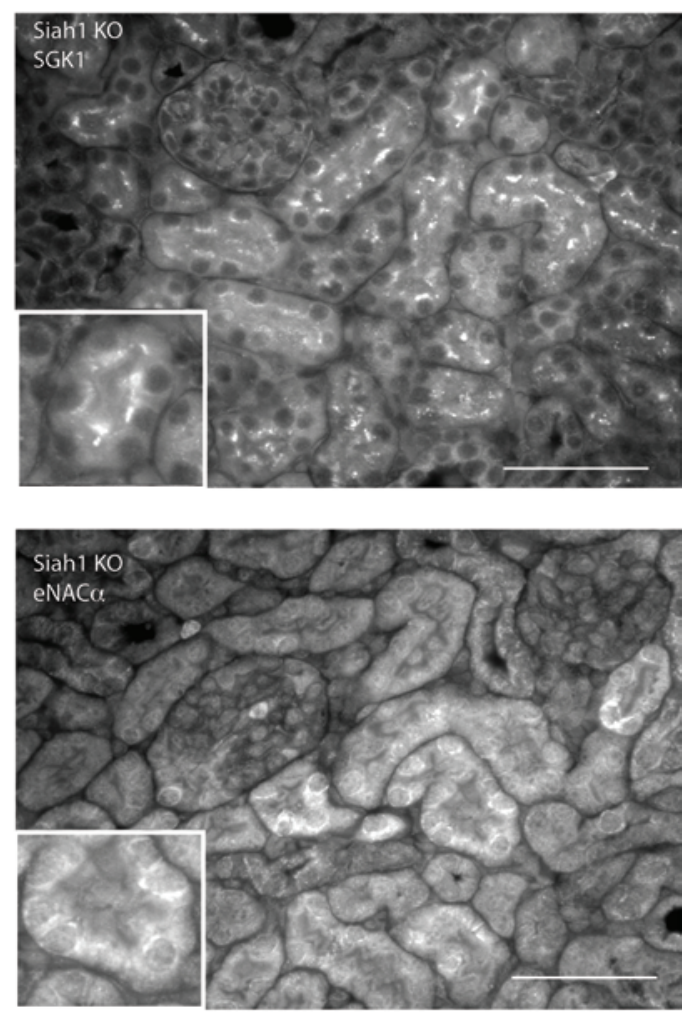

Figure 4. Increased SGK1 and eNAC $\alpha$ expression in kidneys of Siah1a ${ }^{-/-}$mice. (A and B) qPCR analysis of Sgk1 and eNAC $\alpha$ mRNA in the kidneys of WT and Siah1a KO mice $(n=6) .{ }^{*} P<0.05$, compared with WT. (C and D) Staining of SCK1 (C) and eNAC $\alpha$ (D) shown in white from sections of kidneys. Images shown are representative from 4 different mice per group. Scale bar: $50 \mu \mathrm{m}$. Original magnification, $\times 2.5$ (insets). (E) Potassium levels in the plasma of WT and Siah1a KO mice $(n=5) .{ }^{*} P<0.005$, compared with WT. Data are shown as mean \pm SEM. Statistical analysis was performed using $t$ test. 


\section{A}

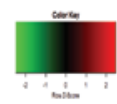

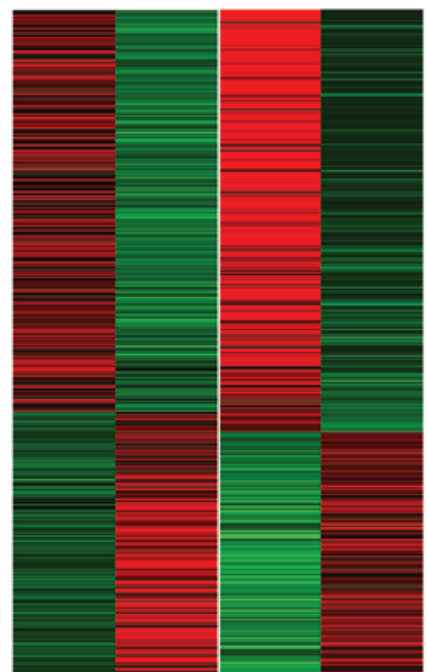

WT Female KO Female WT Male Ko Male
Top Canonical Pathways downregulated in Siah1a KO:

cholesterol biosynthesis

FXR/RXR activation

LPS/IL-1 in RXR Function

tryptophan degradation

stearate biosyntesis

LXR/RXR activation

TR/RXR activation

serotonin degradation

Top Canonical Pathways upregulated in Siah1a KO:

catecholamine biosynthesis

ephrin receptor signaling

Gi signaling

triacylglycerol biosynthesis

glycogen degradation

IGF-1 signaling

April signaling

CAMP signaling

B
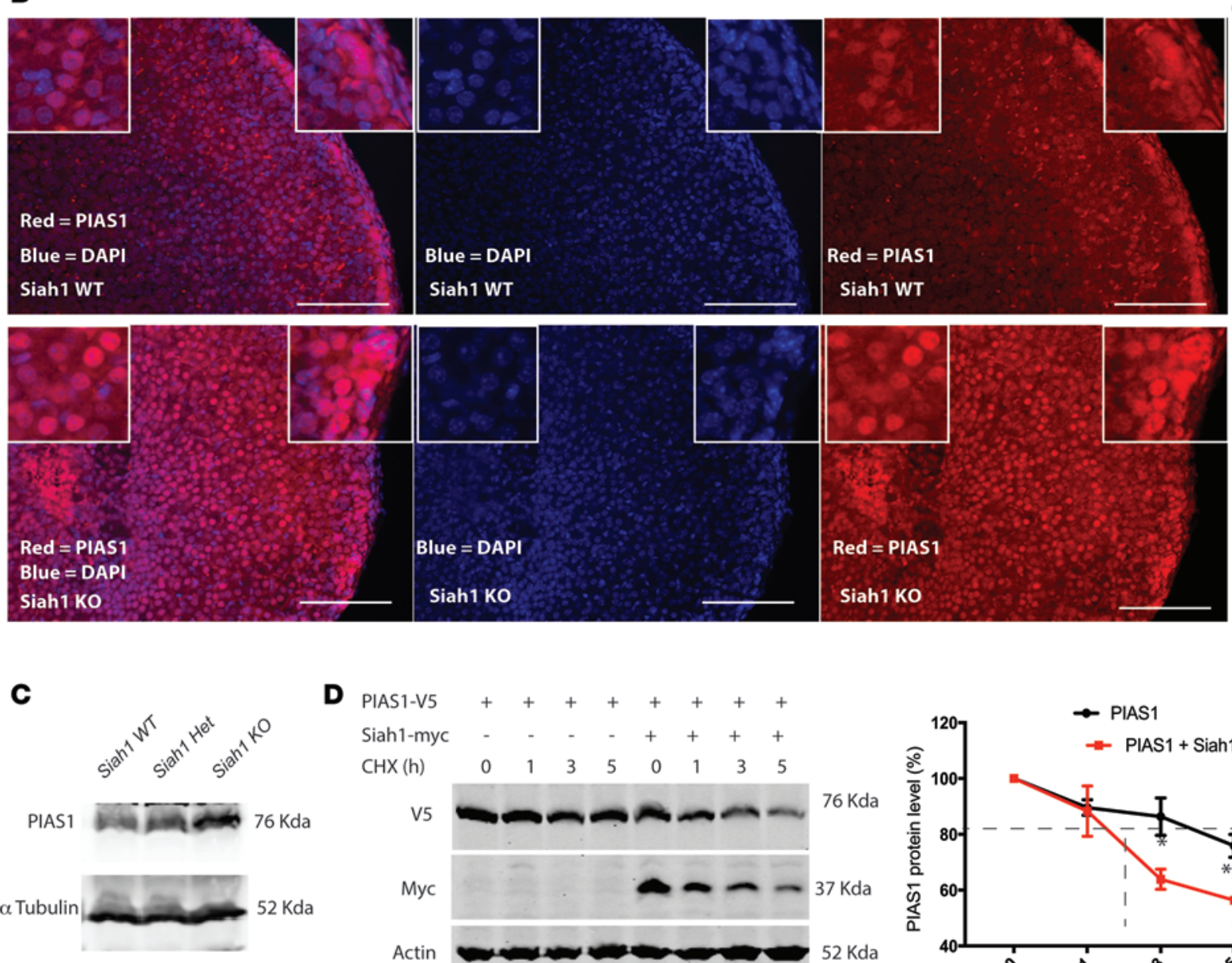

D
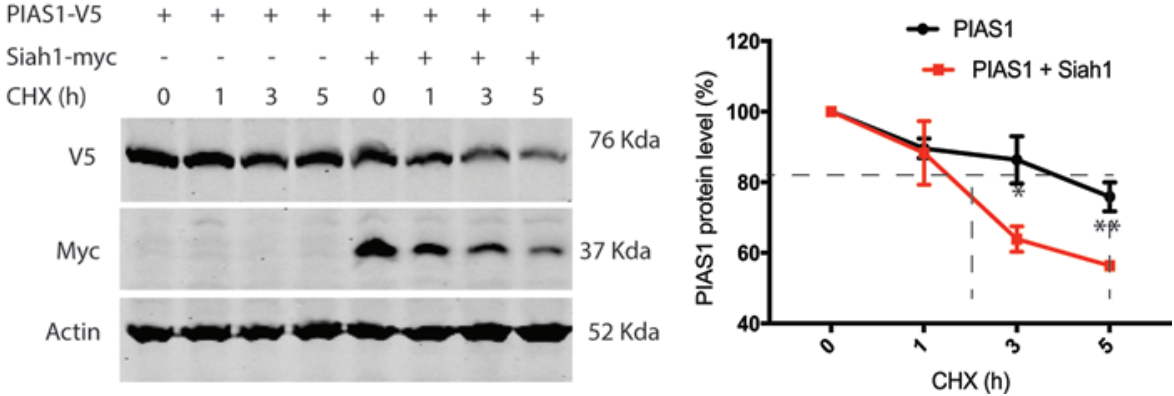

Figure 5. Downregulation of LXR/RXR and FXR/RXR expression and cholesterol biosynthesis correlates with induction of PIAS1 SUMO E3 ligase protein in the adrenal glands of Siah1a-/- mice. (A) Heatmap of RNA-seq data from the adrenal glands of female and male WT and Siah1a KO mice, showing the top canonical pathways upregulated and downregulated in Siah1a KO mice. Selection criteria were $P \leq 0.05$ and $\geq 2$-fold change in expression. (B) PIAS1 staining (red) in sections of adrenal glands from 21-day-old WT and Siah1a KO mice. Scale bar: $100 \mu \mathrm{m}$. Original magnification, $\times 3$ (insets). Images shown are representative of images from 4 different mice per group. (C) Western blot analysis of PIAS1 and tubulin expression in protein lysates of pooled adrenal 
glands from 3 WT, 3 Siah1a ${ }^{+/}$, and 3 Siah1a ${ }^{-/-}$mice. (D) Siah1, PIAS1, and actin expression in 293T cells transfected with PIAS1 alone and PIAS1 plus Siah1. Cells were treated with the protein synthesis inhibitor cycloheximide (CHX) for the indicated times. Dashed lines denote the different degradation rate of PIAS1 alone versus PIAS1 coexpressed with Siah1. The graph shows quantification of PIAS1 degradation from 4 independent experiments. ${ }^{*} P<0.05$, ${ }^{* *} P<$ 0.005 , compared with PIAS1 alone. Data are shown as mean \pm SEM. Statistical analysis was performed using $t$ test.

potential relevance, the rotation axis lies within the $\mathrm{ZnF}-1-\mathrm{ZnF}-2$ linker, at point of contact with the N-terminal end of the helix-turn-helix element containing Ile 251. We thus hypothesize that the I251L mutation affects the conformational control/dynamics of ZnF-2-RING moiety with respect to the SBD during substrate presentation to the UBP machinery. The effect of similar subtle mutations on protein's function has been well established (36). In addition, the effect of the mutation may be enhanced by the combinatorial effect of an effective "double mutant" generated across the dimer axis. These observations provide a testable hypothesis for the strong functional changes elicited by this mutant. To test this, we expressed PIAS1 and the WT or I251L forms of human SIAH1 in 293T cells and analyzed PIAS1 degradation. As expected, PIAS1 half-life was prolonged in cells expressing SIAH1-I251L, compared with SIAH1 WT (40\% of PIAS1 protein was degraded within 5 hours, compared with 2.5 hours, when coexpressed with SIAH1-I251L or SIAH1 WT, respectively; Figure 6B). Likewise, SIAH1-I251L did not affect the stability of $\beta$-catenin, another SIAH1 substrate (Supplemental Figure 5A and ref. 35), confirming that the I251L mutation impaired SIAH1 ubiquitin ligase activity. Although mRNA levels of Axin2 and Lef1, two downstream target genes of the $\beta$-catenin signaling pathway, were unaltered in the adrenal glands of WT and Siah1 $1 a^{-/}$mice (Supplemental Figure 4I), increased nuclear localization of PIAS1 was observed in adrenal glands of Siah1 $1 a^{-/-}$mice (Figure 5B). Consistent with this, immunohistochemical staining of PIAS1 in adrenal gland sections from the patient carrying the SIAH1-I251L mutation also showed an increase in nuclear PIAS1 expression (Figure 6C). Further, the tumor sample of the patient carrying the I251L mutation exhibited lower Siah1 expression, which coincided with an increase in PIAS1 levels (Supplemental Figure 5B). Likewise, an increase in PIAS1 and a decrease in SIAH1 expression were also seen in tumor harboring the SIAH1 R29I mutation, compared with expression in the zona glomerulosa (Figure 6C and Supplemental Figure 5B). This observation prompted us to assess the possible presence of somatic mutation of SIAH1 in respective tumors. Somatic analysis was performed on tumors from patients carrying the I251L and R29I mutations (NM_001006610). A loss of the normal SIAH1 allele was identified in patient harboring the R29I mutation. No mutations or deletion were identified in tumor from the patient harboring the $\mathrm{I} 251 \mathrm{~L}$ mutation, suggesting that the decreased Siah1 protein level could be caused by posttranslational changes. The latter is consistent with the reported decrease in Siah1 mRNA expression $(P<0.0275)$ in patients with PA (GSE8514) (37). Moreover, the analysis of a panel of aldosterone-producing tumors without SIAH1 mutations revealed that 5 of the 8 tumors analyzed exhibited reduced cytoplasmic and increased nuclear SIAH1 expression (data not shown). Of interest, we did not identify notable changes in the activity of the SIAH1 mutant R29I, possibly since the site is proximal to phosphoacceptor sites, which may require select conditions/ phosphorylation for its activity. These results further support the important role of Siah1 signaling in $\mathrm{PA}$ and suggest that SIAH1 expression is altered in aldosterone-producing tumors.

Table 1. Clinical data of the 81 patients included in this study

\begin{tabular}{|c|c|c|}
\hline Age at diagnosis in yr (mean \pm SD) & & \\
\hline Sex & Female & $n=48$ \\
\hline Diagnosis & Combined & $n=21$ \\
\hline \multirow[b]{2}{*}{ Adrenal morphology } & Adrenal adenoma & $n=37$ \\
\hline & Unilateral adrenal hyperplasia & $n=7$ \\
\hline
\end{tabular}


Table 2. Summary of the genetic variants and in silico analysis

\begin{tabular}{|c|c|c|c|c|c|c|c|c|c|c|c|}
\hline \multicolumn{2}{|c|}{ Germline } & \multirow[b]{2}{*}{ Tumor } & \multirow[b]{2}{*}{ Isoform ID } & \multirow{2}{*}{$\begin{array}{c}\text { Frequency in } \\
\text { ExAc }\end{array}$} & & \multirow[b]{2}{*}{ SNP identification } & \multicolumn{5}{|c|}{ In silico analysis ${ }^{A}$} \\
\hline $\begin{array}{l}\text { DNA } \\
\text { change }\end{array}$ & $\begin{array}{l}\text { Protein } \\
\text { change }\end{array}$ & & & & & & (a) & (b) & (c) & (d) & (e) \\
\hline c. $751 \mathrm{~A}>\mathrm{C}$ & p.1251L & No LOH & NM_003031 & $\begin{array}{l}\text { Identified in } \\
\text { this study }\end{array}$ & Missense & - & DC & DC & $P$ & $P$ & M \\
\hline c. $63 \mathrm{G}>\mathrm{A}$ & p.R21R & $N / A^{B}$ & NM_003031 & 0.0002 & Synonymous & rs780812066 & $P(s)$ & $\mathrm{P}$ & $P$ & $\mathrm{~N} / \mathrm{A}$ & $\mathrm{N} / \mathrm{A}$ \\
\hline c. $813 C>T$ & p.G271G & $N / A^{B}$ & NM_003031 & 0.006 & Synonymous & rs35374298 & $D C$ & $\mathrm{P}$ & $\mathrm{P}$ & $\mathrm{N} / \mathrm{A}$ & $\mathrm{N} / \mathrm{A}$ \\
\hline c. $630 G>A$ & p.Q210Q & $N / A^{B}$ & NM_003031 & $\begin{array}{l}\text { Identified in } \\
\text { this study }\end{array}$ & Synonymous & - & DC & $P$ & $P$ & & \\
\hline c.564C $>\mathrm{T}$ & p.H188H & $N / A^{B}$ & NM_003031 & 0.001 & Synonymous & rs183087746 & DC & $\mathrm{P}$ & $\mathrm{P}$ & $\mathrm{N} / \mathrm{A}$ & $\mathrm{N} / \mathrm{A}$ \\
\hline
\end{tabular}

AIn silico analysis was performed using the prediction tools (a) MutationTaster, (b) SIFT, (c) PROVEAN, (d) Polyphen-2, and (e) Mutation Assessor. ${ }^{B}$ No available tissue. ${ }^{C}$ For the nomenclature of this variant the isoform NM_001006610 was used. DC, disease causing; P, polymorphism; M, medium; (s), splice site; LOH; loss of heterozygosity.

\section{Discussion}

In all, using a genetic Siah1 $a^{-/-}$mouse model, we demonstrate a role for Siah1a in adrenal gland development and function. Adrenal glands of Siah1a ${ }^{-/-}$mice exhibit a diminished X-zone, enlarged medulla, and dysregulated zonation of the glomerulosa. The murine X-zone is comparable to the fetal zone of the human adrenal, which regresses with age. Mice carrying an SF-1 mutation, which eliminates its sumoylation, exhibit a persistent fetal X-zone, suggesting that SF1 sumoylation can interfere with the normal maturation of adrenal gland and regression of the X-zone (38). Notably, the Siah1 substrate PIAS1 was shown to be a key activator of SF-1 mediated transcription (33). Thus, the increased expression of PIAS1 in the adrenal gland of Siah1 $1 a^{-1-}$ mice may affect SF1 activity and lead to accelerated X-zone degeneration.

Along with a diminished X-zone, Siah1 $a^{-/-}$adrenal glands exhibit an enlarged medulla and an increased adrenaline level in the plasma. The role of E3 ligases in adrenal medulla development and activity remains still largely unknown, with the exception of mutant VHL, which has been implicated in the pathogenesis of pheochromocytoma (39). Our findings demonstrate that Siah1a can modulate adrenal medulla development and adrenaline production. Mechanisms underlying Siah1a regulation of medulla development, and the implication of its altered expression/mutations in pathological conditions (i.e., pheochromocytomas), remain to be determined.

Loss of Siah1a results in hyperaldosteronism, a disorder strongly associated with hypertension in humans. Changes in aldosterone levels in Siah1a ${ }^{-1-}$ mice were associated with altered $\mathrm{K}^{+}$level, catecholamine biosynthesis, cAMP signaling, retinoic acid signaling, and cholesterol biosynthesis in the adrenal gland. Functional studies established the involvement of the Siah1 substrate PIAS1, which has been implicated in retinoic acid signaling and cholesterol biosynthesis, in mediating the changes observed in the adrenal glands of Siah1 $1 a^{-/-}$mice. Importantly, our observations in the genetic mouse model were validated by the finding that SIAH1 and PIAS1 expression are dysregulated in the adrenal glands of patients with PA. Collectively, these data uncover a role for the Siah1-PIAS1 regulatory axis in aldosterone secretion. In particular, our finding that changes in retinoic acid signaling and cholesterol biosynthesis contribute to adrenal gland dysfunction and hyperaldosteronism raises the possibility that drugs selectively targeting these pathways might offer a new therapeutic modality for PA, a disease that currently lacks effective therapy beyond mineralocorticoid receptor blockade.

\section{Methods}

Cells and transfection. HEK-293T cells were maintained at $37^{\circ} \mathrm{C}$ in Dulbecco's modified Eagle's medium (Invitrogen) with 10\% fetal bovine serum (Millipore) and 1\% penicillin-streptomycin (Invitrogen). Cells were seeded and grown to $60 \%-80 \%$ confluency before transfection with the expression plasmids described below. Transfections were performed using jetPRIME (Polyplus) according to the manufacturer's protocol. 
A

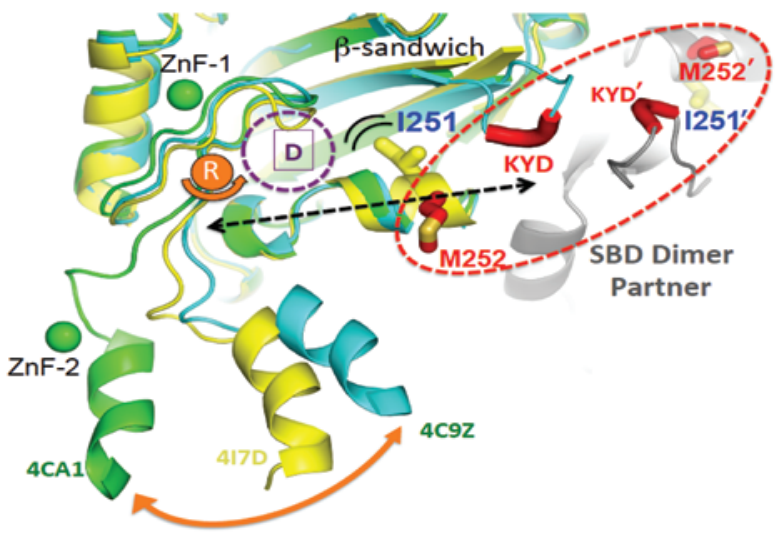

B
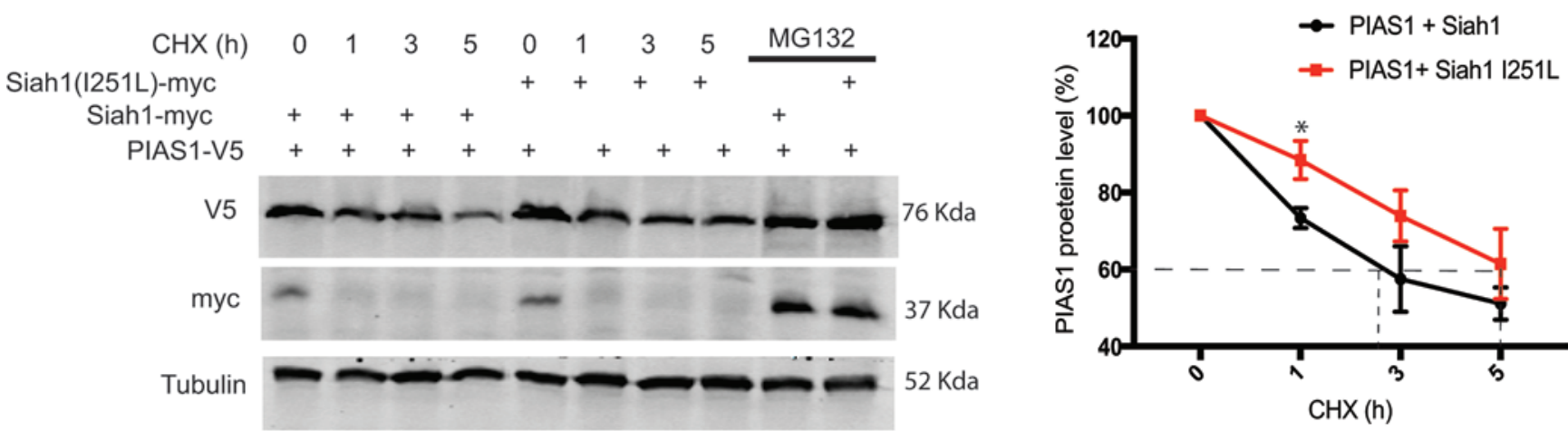

C

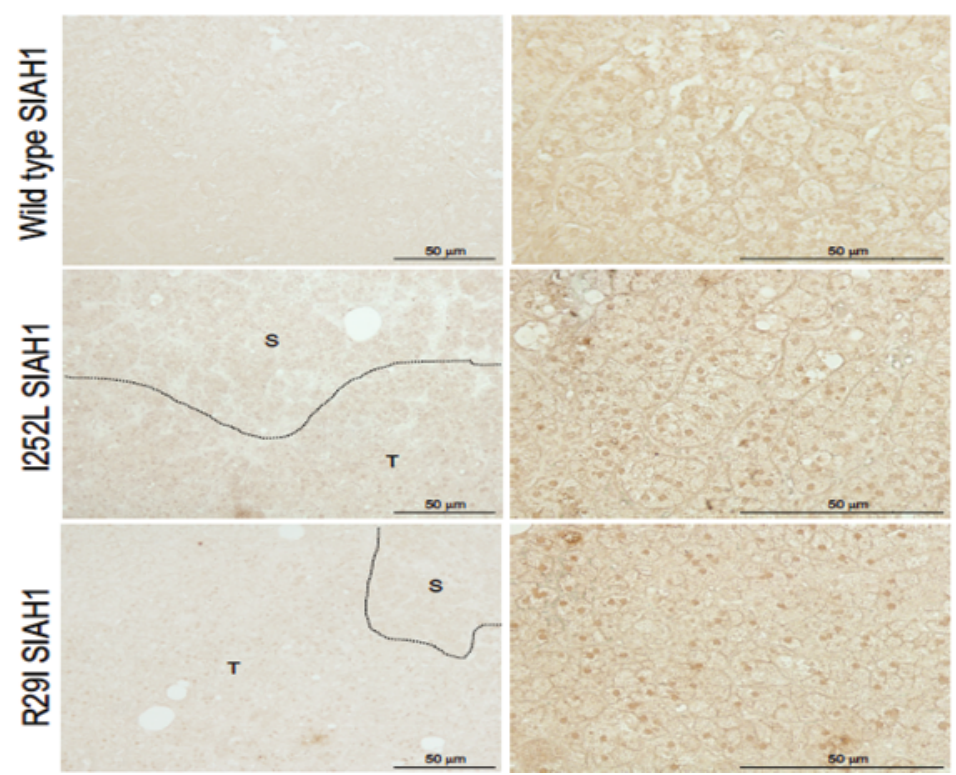

Figure 6. Reduced activity of Siah1 I251L and increased PIAS1 expression in a patient with primary aldosteronism. (A) Cartoon representation of Siah1 surrounding the $1151 \mathrm{~L}$ mutation site (yellow sticks, blue label) that lies on a "helix-turn-helix" element that connects the dimer interface to the linker between the $2 \mathrm{Zn}$ fingers (indicated by dashed black double-headed arrow). lle151 is partly buried but also forms part of a prominent pocket ("D"; brown dashed circle). Loss-of-function mutation sites are colored red, and a red circle emphasizes how they cluster across the SBD dimer interface (part of second SBD is in gray). Three crystal forms are overlaid (see Methods and Results) to demonstrate conformational heterogeneity in the second $\mathrm{Zn}$ finger ( $\mathrm{ZnF}-2$ ). For clarity, $\mathrm{ZnF}-2$ is represented by a single helix (each structure is labeled by its PDB code, see Methods), but the motion is simple rigid-body rotation about the axis labeled " $\mathrm{R}$," circled in orange. The RING domain is N-terminal to $\mathrm{ZnF}-2$, and its structure has not been determined. (B) Western blot analysis of PIAS1 expression in 293T cells transfected with PIAS1-V5 alone or in combination with either Siah1-myc or Siah1-1251L-myc. A cycloheximide (CHX) chase was performed for the indicated times. Blots were probed with antibodies against V5 or myc. Tubulin served as a loading control. Dashed lines denote the different degradation rate of PIAS1 coexpressed with WT Siah1 versus PIAS 1 coexpressed with Siah1-I251L. The graph shows quantification of PIAS1 degradation from 5 independent experiments. ${ }^{*} P<0.05$, compared with 
Siah1 + PIAS1. (C) Immunohistochemical staining of PIAS1 in adrenal gland sections of 2 patients with primary aldosteronism harboring either I251L or R29I. The line represents the delimitation between the surrounding tissue (S) and the tumoral tissue (T). Data are shown as mean \pm SEM. Statistical analysis was performed using $t$ test. Scale bar: $50 \mu \mathrm{m}$

Antibodies and reagents. Antibodies to $\alpha$-tubulin (Santa Cruz, catalog sc-8035), $\beta$-actin (Santa Cruz, cata$\log$ sc-47778), PIAS1 (Cell Signaling for Western blot analysis, catalog 3550; Boster Biological Technology for mouse immunofluorescence, catalog PA2252), Flag (Millipore, catalog F1804), myc (Cell Signaling, catalog 227G), DAB2 (Cell Signaling, catalog 12906), $\beta$-catenin (Abcam, catalog ab6302), MECA 32 (BD biosciences, catalog BDB550563), eNAC $\alpha$ (Bioss antibodies, catalog bs-2957R), SGK1 (Boster biological Technology, catalog PA2184), and V5 (Invitrogen, catalog R96025) were used according to the manufacturers' recommendations. Antibodies to CYP11B1 and CYP11B2 used for staining of mouse adrenal glands were a gift from Celso E. Gomez-Sanchez (University of Mississippi Medical Center, Jackson, Mississippi, USA.) (40). For human samples, the following antibodies were used: anti-SIAH1, catalog PA529682, Thermo Fisher Scientific; anti-PIAS1, catalog ab109388, Abcam; anti-CYP11B2, catalog NBP213891, Novus; and CYP11B1, catalog PA5-63290, ThermoScientific. Cycloheximide was purchased from Millipore and MG132 was purchased from Calbiochem.

Plasmids, cloning, and mutagenesis. Myc-tagged Siah1 and Flag-tagged $\beta$-catenin were cloned as previously described (41). V5-tagged PIAS1 was purchased from DNASU. Mutagenesis of Siah1 was performed using a QuikChange II XL mutagenesis kit (Invitrogen). Sequences of the primers used for mutagenesis are as follow: Siah1-R29I forward, 5'-CCAGCGGCCAGGACAAGGAAGATAAAAGAAATGAGCCGTCAG-3'; Siah1-R29I reverse, 5'-CTGACGGCTCATTTCTTTTATCTTCCTTGTCCTGGCCGCTGG-3'; Siah1-I251L forward, 5'-TGAAGGAATTGCAACAGCCCTTATGAATAGCGACTGTCTA-3'; Siah1-I251L reverse, 5'-TAGACAGTCGCTATTCATAAGGGCTGTTGCAATTCCTTCA-3'.

Western blotting. Adrenal gland tissue was homogenized using RIPA buffer (50 mM Tris- $\mathrm{HCl}, \mathrm{pH} 7.5$, $150 \mathrm{mM} \mathrm{NaCl}, 1 \%$ Triton X-100, 0.1\% SDS, $0.1 \%$ sodium deoxycholate, 1 mM EDTA, 1 mM sodium orthovanadate, $1 \mathrm{mM}$ PMSF, $10 \mu \mathrm{g} / \mathrm{ml}$ aprotinin, and $10 \mu \mathrm{g} / \mathrm{ml}$ leupeptin) and centrifuged. The lysate supernatants were removed, and proteins were resolved by SDS-PAGE and transferred to a nitrocellulose membrane (Osmonics). The membrane was blocked and probed with primary antibodies followed by a secondary antibody. Protein bands were detected using the Odyssey system (Amersham/Bioscience).

qRT-PCR analysis. Total RNA was extracted from adrenal glands using TRIzol (Ambion) and digested with DNase I. cDNA was synthesized using oligo-dT and random hexamer primers for SYBR Green qPCR analysis. H3.3A was amplified as an internal control. All analyses were performed with the adrenal glands from at least 3 mice. The PCR primers were designed using Primer3 and their specificity was checked using BLAST. The PCR products were limited to 100-200 bp. The primer sequences used are as follows: mouse H3.3A, forward, 5'-AAGCAGACTGCCCGCAAAT-3' and reverse, 5'-GGCCTGTAACGATGAGGTTTC-3'; mouse TH, forward, 5'-GTCTCAGAGCAGGATACCAAGC-3' and reverse, 5'-CTCTCCTCGAATACCACAGCC-3'; mouse 20- $\alpha$ HSD, forward, 5'-TCGTCCAGAGTTGGTCAGAC-3' and reverse, 5'-CTTTAGGCAAAAGCTCATTCCCT-3'; mouse Pik3c2g, forward, 5'-TTTAAGGCCGGAGATGATCTTCG-3' and reverse, 5'-AGGGTTACAGCATCAGG-3'; mouse Cyp11b2, forward, 5'-TGGCTGAAGATGATACAGATCCT-3' and reverse, 5'-CACTGTGCCTGAAAATGGGC-3'; mouse Cyp11b1, forward, 5'-CAGATTGTGTTTGTGACGTTGC and reverse, 5'-CGGTTGAAGTACCATTCTGGC-3'; mouse Sgk1, forward, 5'-CGTCCGAACGGGACAACAT-3' and reverse, 5'-GTCCACCGTCCGGTCATAC-3'; mouse Scnn1a, forward, 5'-CCTTCTCCTTGGATAGCCTGG-3' and reverse,5'-CAGACGGCCATCTTGAGTAGC-3'; mouse Axin2, forward, 5'-TGACTCTCCTTCCAGATCCCA-3' and reverse, 5'-TGCCCACACTAGGCTGACA-3'; mouse Lef1, forward, 5'-TGTTTATCCCATCACGGGTGG-3' and reverse, 5'-CATGGAAGTGTCGCCTGACAG-3'; mouse Ppara, forward, 5'-AACATCGAGTGTCGAATATGTGG-3' and reverse, 5'-CCGAATAGTTCGCCGAAAGAA-3'; mouse ApoE, forward, 5'-CTCCCAAGTCACACAAGAACTG-3' and reverse, 5'-CCAGCTCCTTTTTGTAAGCCTTT-3'; mouse Hmgcr forward, 5'-TGTTCACCGGCAACAACAAGA-3' and reverse, 5'-CCGCGTTATCGTCAGGATGA-3'; mouse Pmvk, forward, 5'-AAAATCCGGGAAGGACTTCGT-3' and reverse, 5'-AGAGCACAGATGTTACCTCCA-3'; mouse Dps, forward, 5'-GGAGGTCCTAGAGTACAATGCC-3' and reverse, 5'-AAGCCTGGAGCAGTTCTACAC-3'; mouse Lss, forward 5'-CTCCAGAATGAGTTGGGTCGG-3' and reverse, 5'-CGCTTTTGGTAAGTCCGTGAAA-3'; mouse Scarb1, forward, 5'-TTTGGAGTGGTAGTAAAAAGGGC-3' and reverse, 5'-TGACATCAGGGACTCAGAGTAG-3'; mouse Camk2d, forward, 5'-GATGG- 
GGTAAAGGAGTCAACTG-3' and reverse, 5'-CATTGTGGCATACAGCGACA-3'; mouse Rgs2, forward 5'-GAGAAAATGAAGCGGACACTCT-3' and reverse, 5'-GCAGCCAGCCCATATTTACTG-3'; mouse Pias1, forward, 5'-ACGCAAACACGAACTTCTTACA-3' and reverse, 5'-TCCGCAGGCGTCATAATTTTC-3'; mouse Siah1a, forward, 5'-GCTGAAAATTTTGCATATCG-3' and reverse, 5'-CCAGGAAAGTTTTAGGTTGG-3'; mouse $\beta$-catenin, forward, 5'-ATGGAGCCGGACAGAAAAGC-3' and reverse, 5'-TGGGAGGTGTCAACATCTTCTT-3'; mouse Vegfa, forward, 5'-GCACATAGAGAGAATGAGCTTCC-3' and reverse, 5'-CTCCGCTCTGAACAAGGCT-3'; mouse enac $\beta$, forward, 5'-GGCCCAGGCTACACCTAGA-3' and reverse, 5'-AGCAGCGTAAGCAGGAACC-3'; mouse enac $\gamma$, forward, 5'-GCACCGACCATTAAG-3' and reverse, 5'-GCGTGAACGCAATCCACAAC-3'.

Plasma analysis. Plasma $\mathrm{K}^{+}$levels were measured using a VetScan VS2 analyzer (Abaxis) according to the manufacturer's protocol. Plasma aldosterone, corticosterone, and ACTH were measured using an ELISA kit (Enzo Life Sciences for aldosterone and corticosterone, Biotechnologies System for ACTH) according to the manufacturer's protocol. Renin activity was measured in plasma using the Sensolyte 520 mouse renin assay kit (Anaspec) following the manufacturer's recommendations. Plasma adrenaline and noradrenaline were measured using BI-CAT ELISA (DLD) according to the manufacturer's protocol.

ACTH administration. Mice were injected intraperitoneally with $100 \mu \mathrm{g} / \mathrm{Kg}$ ACTH (1-24) (VWR international). Blood was collected 1 hour after administration.

Histology. Adrenal glands were fixed overnight in Z-fix (buffered zinc formalin fixative, Anatech), washed twice with PBS, and processed for paraffin embedding. Paraffin blocks were sectioned into 5- $\mu \mathrm{m}$ slices and stained with H\&E.

Adrenal zonation measurement. For X-zone measurement, images of $\mathrm{H} \& \mathrm{E}$-stained samples were acquired at the same magnification $(\times 4)$ and processed in Image J64 software. Adrenal widths were then measured. For medulla measurement, medulla and total area of adrenal gland were measured in Image J64 software (NIH).

Immunofluorescence microscopy of mouse tissue. Sections of adrenal glands or kidneys were deparaffinized, rehydrated, washed in PBS, and blocked with Dako protein block for 30 minutes at room temperature. Antigen retrieval was performed in a pressure cooker (Decloaking chamber, Biocare Medical) in citrate buffer ( $\mathrm{pH}$ 6.0) for CYP11B1, CYP11B2, DAB2, $\beta$-catenin, SGK1, and PIAS1 and in high pH for eNAC $\alpha$. Immunostaining was performed by incubating the sections overnight at $4^{\circ} \mathrm{C}$ with antibodies in Dako antibody diluent, whereas 20- $\alpha$ HSD (1:500, Antibody Research) was diluted in 1\% BSA/PBS for immunostaining. Alexa Fluor 488- or Alexa Fluor 594-conjugated secondary antibodies were added for 1 hour at room temperature (1:400, Molecular Probes), and nuclei were counterstained using SlowFade Gold Antifade reagent (Vector) with DAPI (Vector).

Oil Red $O$ staining. Lipid staining was performed on cryosections incubated in 1,2 propanediol for 1 minute and in Oil Red $\mathrm{O}$ (Millipore) at $60^{\circ} \mathrm{C}$ for 10 minutes.

In situ hybridization. RNAscope in situ hybridization was performed using a mouse Siah1a probe (Advanced Cell Diagnostics), according to the manufacturer's protocols.

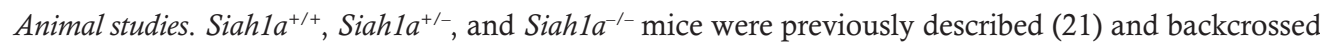
to 129 sv mice for 10 generations.

RNA-seq. PolyA RNA was isolated using the NEBNext Poly(A) mRNA Magnetic Isolation Module, and barcoded libraries were made using the NEBNext Ultra Directional RNA Library Prep Kit for Illumina (NEB). Libraries were pooled and single-end sequenced (1X75) on an Illumina NextSeq 500 using the High Output V2 kit (Illumina). The raw reads (fastq files) were aligned to mouse reference genome using TopHat version 2.013 (http://www.ncbi.nlm.nih.gov/pubmed/19289445) with transcript annotations and the "-no-novel-juncs" option. Ambiguous reads that mapped to more than one region in the genome and reads with MAPQ score less than 10 were removed.

The mouse reference genome $(\mathrm{mm} 10)$ and corresponding transcript annotation downloaded from UCSC were used as a reference genome for mRNA transcript quantification. Transcript quantification was performed using the Partek Genomics Suite (version 6.4, Partek), and the raw read counts and normalized read counts (reads per kilobase per million mapped reads [RPKM]; http://www.ncbi.nlm.nih.gov/ pubmed/18516045) were obtained.

The raw count information for all mRNA transcripts was first filtered. Transcripts with poor read counts in all samples were removed from further analysis. The remaining transcripts were analyzed using generalized linear model approaches implemented in the BioConductor edgeR package (http://www. ncbi.nlm.nih.gov/pubmed/22287627) to detect the differential expression between different conditions. 
For transcripts detected in at least one sample $(\mathrm{RPKM}>1)$, significant differential expression was considered for transcripts with $>2$-fold change and $P<0.05$.

Genetic analysis of SIAH1 gene in peripheral DNA samples. A total of 81 patients with PA that were diagnosed with standard criteria (2) evaluated at the National Institutes of Health Clinical Research Center were included in this study.

DNA was extracted from blood leukocytes according to the manufacturer's protocol (QIAGEN). Specific amplification of the coding region of the SIAH1 gene (16q12.1) in peripheral DNA of 81 patients with PA was performed using the following set of primers: 5'-CGCGAATTTGGGTCTTCTGT-3' and 5'- AAACGTCTCGATTCTGCTGC-3' (for the first exon); 5'-TTTTTCTTCCTCTTTTGCTCAG-3' and 5'-ACTGCATCATCACCCAGTCA-3', and 5'-CCCTGGTGCTTCCTGTAAAT-3' and 5'-CAGCCTTTCTTTTTATTTACCTTC-3' (for the second exon, overlapping pairs). PCR products were purified (ExoSAP-IT reagent; Affimetrix-USB) and sequenced bidirectionally using the same primers (ABI 3500XL; Applied Biosystems).

In silico analysis. In silico analysis to predict the pathogenicity and evolutionary conservation of the missense and synonymous variants found in the SIAH1 gene was performed using the MutationTaster (http:// www.mutationtaster.org/) prediction tool.

Immunohistochemistry of human adrenal glands. Five-micrometer sections of paraffin-embedded tissue blocks were deparaffinized in Histo-Clear (Nationals Diagnostics) and rehydrated through a series of ethanol dilutions. Antigen was unmasked by incubation for 20 minutes in a boiling Tween/citrate buffer ( $\mathrm{pH}$ 6 ), and the slides were blocked with $1 \%$ bovine serum albumin in PBS. Sections were incubated overnight at $4^{\circ} \mathrm{C}$ with primary antibody (1:200 anti-SIAH1, PA5-29682, Thermo Fisher Scientific; 1:200 anti-PIAS1, ab109388, Abcam; 1:100 anti-CYP11B2, NBP2-13891, Novus; or 1:100 CYP11B1, PA5-63290, ThermoScientific), washed, and incubated with a biotin-coupled anti-rabbit secondary antibody (Jackson ImmunoResearch). After washing, the sections were incubated with streptavidin-coupled horseradish peroxidase for 30 minutes at room temperature, and peroxidase activity was detected with 3,3'-diaminobenzidine tetrahydrochloride (SJ-4105; Vector Labs).

Structural analysis of Siah1. Three different crystal structures of SIAH1 were overlaid: 2 high-resolution native structures (PDB codes 4CA1 and 4C9Z) and 1 structure with a PHYL-like peptide bound (4I7D) $(35,42)$. Overall RMS differences were initially in the 0.6-0.7 $\AA$ range but clearly showed divergence of the $\mathrm{ZnF}-2$ domain. Structures were therefore realigned on the SBD and ZnF-1 fragments only, giving RMS differences in the $0.2-0.3 \AA$ range (i.e., within the estimated error based on random coordinate errors within individual structures). Structural comparisons (using this latter overlay) and Figure 4A were generated using PYMOL (Copyright Schrödinger LLC).

Statistics. Statistics were calculated using GraphPad Prism 7. Data were analyzed by 2-tailed Student's $t$ test. $P<0.05$ was considered statistically significant.

Study approval. All animal experiments were performed according to protocols approved by Institutional Animal Care and Use Committee at Sanford Burnham Prebys Medical Discovery Institute. The human research protocol (00-CH-0160) was approved by the Institutional Review Boards at the NIH. All patients provided informed consent.

\section{Author contributions}

MS, CAS, and ZAR designed the study. MS, AB, NS, AG, and GG performed the experiments. BJ, RCL, JLL, MS, ZAR, CAS, and JGVM analyzed the data. MS, CAS, and ZAR wrote the manuscript.

\section{Acknowledgments}

We thank David Bowtell (PeterMac, Melbourne, Australia) for providing us with the Siah1a KO mice. We also thank Celso Gomez-Sanchez for providing the antibodies against CYP11B1 and CYP11B2, the histology core at Sanford Burnham Prebys Medical Discovery Institute for pathology histology services (supported by NCI Cancer Center Support Grant P30 CA30199) and for sectioning the tissues, Stephanie Myers for technical support, and members of the Ronai lab for helpful discussions. Support by National Cancer Institute grant (R35 CA197465-01) is gratefully acknowledged.

Address correspondence to: Ze'ev Ronai, Sanford Burnham Prebys Medical Discovery Institute, 10901 N. Torrey Pines Rd., La Jolla, California 92037, USA. Phone: 858.646.3185; Email: zeev@ronailab.net; 
Technion Integrated Cancer Center, Technion, 1 Effron Street, Haifa 3109602, Israel. Phone: 04.829.5447; Email: zeev@ronailab.net. Or to: Marzia Scortegagna, Sanford Burnham Prebys Medical Discovery Institute, 10901 N. Torrey Pines Rd, La Jolla, California 92037, USA. Phone: 858.646.3100, ext. 4207; Email: mscortegagna@sbpdiscovery.org.

1. Funder JW. The genetic basis of primary aldosteronism. Curr Hypertens Rep. 2012;14(2):120-124.

2. Funder JW, et al. Case detection, diagnosis, and treatment of patients with primary aldosteronism: an endocrine society clinical practice guideline. J Clin Endocrinol Metab. 2008;93(9):3266-3281.

3. Young WF. Primary aldosteronism: renaissance of a syndrome. Clin Endocrinol (Oxf). 2007;66(5):607-618.

4. Howard B, et al. Integrated analysis of genome-wide methylation and gene expression shows epigenetic regulation of CYP11B2 in aldosteronomas. J Clin Endocrinol Metab. 2014;99(3):E536-E543.

5. Yoshii Y, et al. Hypomethylation of CYP11B2 in aldosterone-producing adenoma. Hypertension. 2016;68(6):1432-1437.

6. Aguiar M, Masse R, Gibbs BF. Regulation of cytochrome P450 by posttranslational modification. Drug Metab Rev. 2005;37(2):379-404.

7. Knutson TP, Lange CA. Dynamic regulation of steroid hormone receptor transcriptional activity by reversible SUMOylation. Vitam Horm. 2013;93:227-261.

8. Paz C, et al. Role of protein phosphorylation and tyrosine phosphatases in the adrenal regulation of steroid synthesis and mitochondrial function. Front Endocrinol (Lausanne). 2016;7:60.

9. Weigel NL, Moore NL. Steroid receptor phosphorylation: a key modulator of multiple receptor functions. Mol Endocrinol. 2007;21(10):2311-2319.

10. Talamillo A, Martín D, Hjerpe R, Sánchez J, Barrio R. SUMO and ubiquitin modifications during steroid hormone synthesis and function. Biochem Soc Trans. 2010;38(pt 1):54-59.

11. Ehrlund A, et al. E3 ubiquitin ligase RNF31 cooperates with DAX-1 in transcriptional repression of steroidogenesis. Mol Cell Biol. 2009;29(8):2230-2242.

12. Komatsu T, et al. Small ubiquitin-like modifier 1 (SUMO-1) modification of the synergy control motif of Ad4 binding protein/steroidogenic factor 1 (Ad4BP/SF-1) regulates synergistic transcription between Ad4BP/SF-1 and Sox9. Mol Endocrinol. 2004;18(10):2451-2462.

13. Netzer C, Bohlander SK, Rieger L, Müller S, Kohlhase J. Interaction of the developmental regulator SALL1 with UBE2I and SUMO-1. Biochem Biophys Res Commun. 2002;296(4):870-876.

14. Tempé D, Casas M, Karaz S, Blanchet-Tournier MF, Concordet JP. Multisite protein kinase A and glycogen synthase kinase 3beta phosphorylation leads to Gli3 ubiquitination by SCFbetaTrCP. Mol Cell Biol. 2006;26(11):4316-4326.

15. Della NG, Senior PV, Bowtell DD. Isolation and characterisation of murine homologues of the Drosophila seven in absentia gene (sina). Development. 1993;117(4):1333-1343.

16. Nakayama K, et al. Siah2 regulates stability of prolyl-hydroxylases, controls HIF1alpha abundance, and modulates physiological responses to hypoxia. Cell. 2004;117(7):941-952.

17. Scortegagna M, et al. Fine tuning of the UPR by the ubiquitin ligases Siah1/2. PLoS Genet. 2014;10(5):e1004348.

18. Kim H, Claps G, Möller A, Bowtell D, Lu X, Ronai ZA. Siah2 regulates tight junction integrity and cell polarity through control of ASPP2 stability. Oncogene. 2014;33(15):2004-2010.

19. Kim H, et al. Fine-tuning of Drp1/Fis1 availability by AKAP121/Siah2 regulates mitochondrial adaptation to hypoxia. Mol Cell. 2011;44(4):532-544.

20. Nadeau RJ, Toher JL, Yang X, Kovalenko D, Friesel R. Regulation of Sprouty2 stability by mammalian Seven-in-Absentia homolog 2. J Cell Biochem. 2007;100(1):151-160.

21. Dickins RA, et al. The ubiquitin ligase component Siah1a is required for completion of meiosis I in male mice. Mol Cell Biol. 2002;22(7):2294-2303

22. Frew IJ, et al. Osteopenia in Siah1a mutant mice. J Biol Chem. 2004;279(28):29583-29588.

23. Romero DG, et al. Disabled-2 is expressed in adrenal zona glomerulosa and is involved in aldosterone secretion. Endocrinology. 2007;148(6):2644-2652.

24. Gumz ML, et al. The circadian clock protein Period 1 regulates expression of the renal epithelial sodium channel in mice. J Clin Invest. 2009;119(8):2423-2434

25. Hou J, Speirs HJ, Seckl JR, Brown RW. Sgk1 gene expression in kidney and its regulation by aldosterone: spatio-temporal heterogeneity and quantitative analysis. J Am Soc Nephrol. 2002;13(5):1190-1198.

26. Debonneville $\mathrm{C}$, et al. Phosphorylation of Nedd4-2 by Sgk1 regulates epithelial $\mathrm{Na}(+)$ channel cell surface expression. $E M B O J$. 2001;20(24):7052-7059.

27. Cummins CL, et al. Liver X receptors regulate adrenal cholesterol balance. J Clin Invest. 2006;116(7):1902-1912.

28. Depaux A, Regnier-Ricard F, Germani A, Varin-Blank N. A crosstalk between hSiah2 and Pias E3-ligases modulates Pias-dependent activation. Oncogene. 2007;26(46):6665-6676.

29. Lee JH, Kim H, Park SJ, Woo JH, Joe EH, Jou I. Small heterodimer partner SHP mediates liver X receptor (LXR)-dependent suppression of inflammatory signaling by promoting LXR SUMOylation specifically in astrocytes. Sci Signal. 2016;9(439):ra78.

30. Zhang Y, et al. A role for protein inhibitor of activated STAT1 (PIAS1) in lipogenic regulation through SUMOylation-independent suppression of liver X receptors. J Biol Chem. 2012;287(45):37973-37985.

31. Kurihara I, et al. Ubc 9 and protein inhibitor of activated STAT 1 activate chicken ovalbumin upstream promoter-transcription factor I-mediated human CYP11B2 gene transcription. J Biol Chem. 2005;280(8):6721-6730.

32. Shibata H, et al. COUP-TF and transcriptional co-regulators in adrenal steroidogenesis. Endocr Res. 2004;30(4):795-801

33. Suda N, et al. Coactivation of SF-1-mediated transcription of steroidogenic enzymes by Ubc9 and PIAS1. Endocrinology. 2011;152(6):2266-2277. 
34. Anupriya G, Roopa K, Basappa S, Chong YS, Annamalai L. Homology modeling and in silico screening of inhibitors for the substrate binding domain of human Siah2: implications for hypoxia-induced cancers. J Mol Model. 2011;17(12):3325-3332.

35. Santelli E, et al. Structural analysis of Siah1-Siah-interacting protein interactions and insights into the assembly of an E3 ligase multiprotein complex. J Biol Chem. 2005;280(40):342s78-34287.

36. Baase WA, Liu L, Tronrud DE, Matthews BW. Lessons from the lysozyme of phage T4. Protein Sci. 2010;19(4):631-641.

37. Ye P, Mariniello B, Mantero F, Shibata H, Rainey WE. G-protein-coupled receptors in aldosterone-producing adenomas: a potential cause of hyperaldosteronism. J Endocrinol. 2007;195(1):39-48.

38. Lee FY, et al. Eliminating SF-1 (NR5A1) sumoylation in vivo results in ectopic hedgehog signaling and disruption of endocrine development. Dev Cell. 2011;21(2):315-327.

39. Crespigio J, et al. Von Hippel-Lindau disease: a single gene, several hereditary tumors [published online ahead of print June 6, 2017]. J Endocrinol Invest. doi: 10.1007/s40618-017-0683-1.

40. Gomez-Sanchez CE, et al. Development of monoclonal antibodies against human CYP11B1 and CYP11B2. Mol Cell Endocrinol. 2014;383(1-2):111-117.

41. Matsuzawa SI, Reed JC. Siah-1, SIP, and Ebi collaborate in a novel pathway for beta-catenin degradation linked to p53 responses. Mol Cell. 2001;7(5):915-926.

42. Stebbins JL, et al. Structure-based design of covalent Siah inhibitors. Chem Biol. 2013;20(8):973-982. 